Issued by Sandia National Laboratories, operated for the United States Department of Energy by Sandia Corporation.

NOTICE: This report was prepared as an account of work sponsored by an agency of the United States Government. Neither the United States Government, nor any agency thereof, nor any of their employees, nor any of their contractors, subcontractors, or their employees, make any warranty, express or implied, or assume any legal liability or responsibility for the accuracy, completeness, or usefulness of any information, apparatus, product, or process disclosed, or represent that its use would not infringe privately owned rights. Reference herein to any specific commercial product, process, or service by trade name, trademark, manufacturer, or otherwise, does not necessarily constitute or imply its endorsement, recommendation, or favoring by the United States Government, any agency thereof, or any of their contractors or subcontractors. The views and opinions expressed herein do not necessarily state or reflect those of the United States Government, any agency thereof, or any of their contractors.

Printed in the United States of America. This report has been reproduced directly from the best available copy.

Available to DOE and DOE contractors from Office of Scientific and Technical Information P.O. Box 62

Oak Ridge, TN 37831

Prices available from (703) 605-6000

Web site: http://www.ntis.gov/ordering.htm

Available to the public from

National Technical Information Service

U.S. Department of Commerce

5285 Port Royal Rd

Springfield, VA 22161

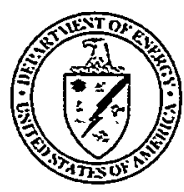




\section{DISCLAIMER}

Portions of this document may be illegible in electronic image products. Images are produced from the best available original document. 
SAND2000-0025

Unlimited Release

Printed January 2000

\title{
LDRD Final Report Backside Localization of Open and Shorted IC Interconnections LDRD PROJECT (FY98 and FY99)
}

\author{
Edward I. Cole Jr., Paiboon Tangyunyong, and Daniel L. Barton \\ Failure Analysis Department \\ David A. Benson \\ Advanced Packaging Department \\ Sandia National Laboratories \\ P.O. Box 5800 \\ Albuquerque, NM 87185-1081
}

\begin{abstract}
Two new failure analysis techniques have been developed for backside and front side localization of open and shorted interconnections on ICs. These scanning optical microscopy techniques take advantage of the interactions between IC defects and localized heating using a focused infrared laser $(\lambda=1340 \mathrm{~nm})$. Images are produced by monitoring the voltage changes across a constant current supply used to power the IC as the laser beam is scanned across the sample. The methods utilize the Seebeck Effect to localize open interconnections and Thermally-Induced Voltage Alteration (TIVA) to detects shorts. Initial investigations demonstrated the feasibility of TIVA and Seebeck Effect Imaging (SED). Subsequent improvements have greatly increased the sensitivity of the TIVA/SEI system, reducing the acquisition times by more than $20 \mathrm{X}$ and localizing previously unobserved defects. The interaction physics describing the signal generation process and several examples demonstrating the localization of opens and shorts are described. Operational guidelines and limitations are also discussed. The system improvements, non-linear response of IC defects to heating, modeling of laser heating and examples using the improved system for failure analysis are presented.
\end{abstract}




\section{ACKNOWLEDGMENTS}

The authors thank Steve Kirch of Intel Corporation, Richard Anderson, Ann Campbell, Jerry Soden, and Jon Weiss of Sandia National Laboratories, and Charles Hawkins of the University of New Mexico for their careful review of the report. The authors would like to thank Alejandro Pimentel for FIB cross sectioning of the test structure. 
ACKNOWLEDGMENTS ............................................................................................. 2

NOMENCLATURE.......................................................................................................... 4

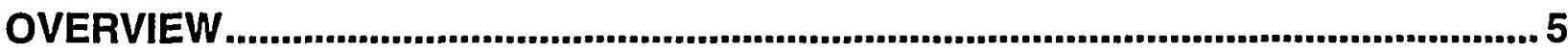

TECHNICAL ACCOMPLISHMENTS ........................................................................ 6

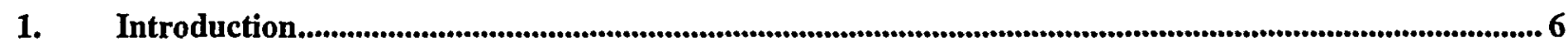

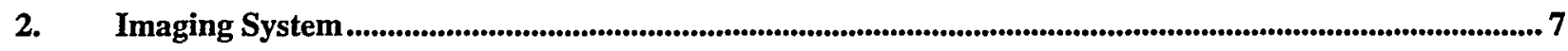

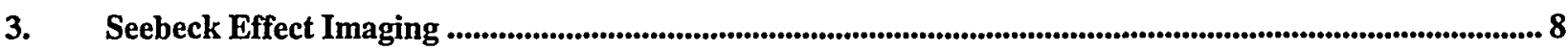

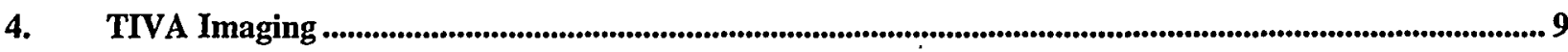

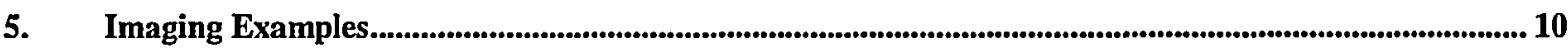

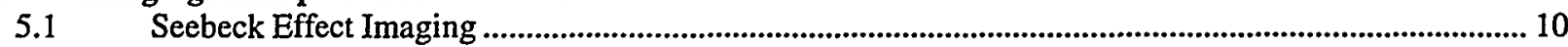

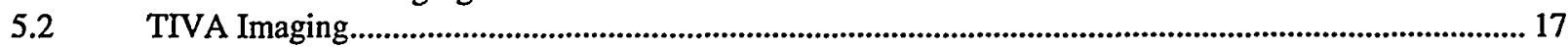

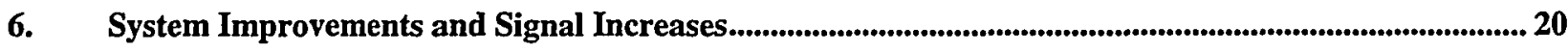

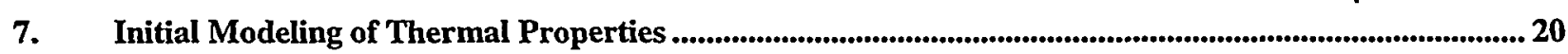

8. Imaging Results with the Improved System ....................................................................................................... 22

9 Extended Thermal Modeling and TIVA Analysis of a Polysilicon-Metal Test Structure ........................ 26

9.1 Experimental Procedures for Analysis of the Test Structure ............................................................................2 26

$9.2 \quad$ Thermal Modeling and Simulations of the Test Structure ........................................................................... 27

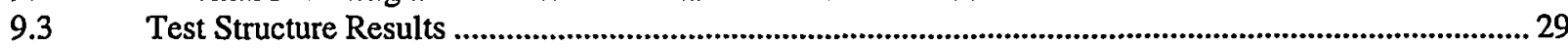

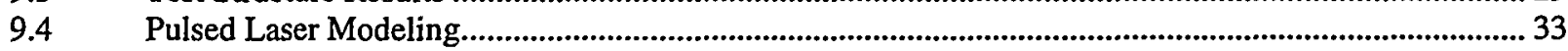

10. Conclusions

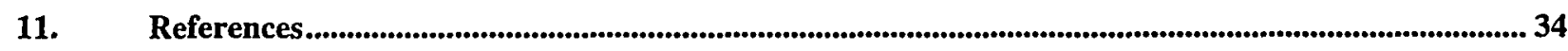

EVALUATION OF PROGRESS TOWARDS GOALS................................................. 36

PATENTS AND INTELLECTUAL PROPERTY .......................................................... 40

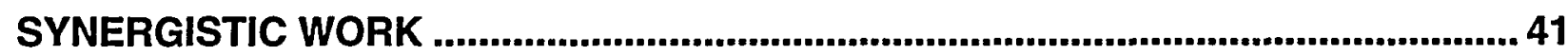

POTENTIAL FUTURE WORK ........................................................................................ 41

BENEFITS TO DOE DEFENSE PROGRAMS....................................................................41 4

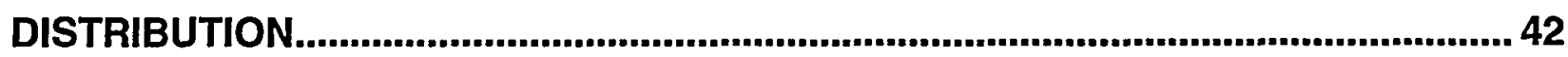




\section{NOMENCLATURE}

ASIC - application specific integrated circuit

CIVA - charge-induced voltage alteration

CMOS - complimentary metal oxide semiconductor

FIB - focused ion beam

FMI - fluorescent microthermal imaging

IC - integrated circuit

LIVA - light-induced voltage alteration

OBIRCH - optical beam induced resistance change

SEI - Seebeck effect imaging

SL - short localization

SOM - scanning optical microscope

SRAM - static random access memory

TAS - thermal analysis software

TIVA - thermally-induced voltage alteration 


\section{OVERVIEW}

This report describes the accomplishments performed under the Sandia National Laboratories LDRD project titled "Backside Localization of Open and Shorted IC (Integrated Circuit) Interconnections." The complexities of today's ICs which employ multiple levels of metallization and flip-chip packaging make backside analysis tools a necessity. This LDRD addresses the need for the development of tools that can quickly and with high sensitivity localize shorted and open interconnections from the backside of an IC. Such tools can be used in failure analysis, design debugging, and yield improvement. Localized laser heating was chosen because of previously demonstrated capabilities for backside analysis. These capabilities have been coupled with Sandia developed technologies for ultra-high sensitivity detection and localization of IC internal defects. The Seebeck Effect Imaging (SEI) technique developed under the LDRD has shown for the first time that open interconnections can be localized directly from the backside of an IC. The Thermally-Induced Voltage Alteration (TIVA) method has localized shorted interconnections that were previously unobservable with conventional failure analysis tools. TIVA in particular has shown applicability to a wide range of IC technologies, including state-of-the-art, multimillion transistor devices.

Our experimental approach and results are described in detail in the report. A major breakthrough is the non-linear, almost exponential increase in TIVA and SEI sensitivity with increasing laser power. We also describe thermal modeling analyses on various structures showing how thermal gradients are distributed on different IC structures.

We evaluate our progress towards the milestones set at the beginning of the LDRD project. We have met or exceeded most of the goals originally generated. One goal that was not successfully met was an improvement in signal sensitivity with laser pulsing synchronized with a lock-in amplifier. We address why we believe this was not successful. Fortunately, the exponential increases in signal sensitivity with laser power mitigated the need for lock-in amplification.

We also describe the impact this LDRD project has had. The TIVA and SEI analysis methods have become standard tools in Sandia's Failure Analysis Laboratory, successfully localizing a number of IC defects. Three papers on the TIVA/SEI techniques have been published (one invited) and the technique has been described in three seminars and workshops. One paper on the modeling aspects of TIVA imaging is pending publication. A patent has been filed for TIVA/SEI and licensing of an IC manufacturer to implement the technique in their laboratory is nearing completion. Several other IC manufacturers have expressed an interest in licensing the TIVA/SEI technology for their laboratories. There has been significant interest in commercialization of a turn-key system for performing TIVA/SEI and initial inquires have been made to Sandia by several possible TIVA/SEI system suppliers. We are presently in the second year of at $\$ 300 \mathrm{~K}$ per year CRADA that has been a direct outgrowth of the TIVA/SEI LDRD. The benefit to DOE Defense Programs is the improved IC analysis capabilities which may be applied to stockpile support and development of new DP systems. 


\section{TECHNICAL ACCOMPLISHMENTS}

\section{Introduction}

Open and short circuited interconnections are major IC yield and reliability problems and will increase in importance as the number of interconnection levels and length of interconnections continue to increase. The ability to localize the defects responsible for various failure modes is critical in diagnosing IC failures and implementing corrective action. Today's ICs employ multiple levels of metal that obscure lower conductor levels and flip-chip packaging that make IC front side examination techniques either difficult or impossible to apply. These problems have driven the development and use of backside IC analysis techniques. Unfortunately, the present suite of backside methods is not totally effective in localizing open and shorted conductors. Existing methods can be time consuming, yield a great deal of superfluous information, and provide only indirect evidence of open and short circuit defects.

One common aspect of many backside IC analysis techniques is the use of infrared light because of Si's relative transparency at infrared wavelengths. Backside optical microscopy, photon emission microscopy [1,2], and transistor logic state mapping [3,4] use infrared light to either stimulate the IC or monitor reflected/transmitted signals. Backside Optical Beam Induced Resistance Change (OBIRCH) [5] has been shown recently to localize shorted conductors, but the detection sensitivity is limited.

To overcome the limitations of existing techniques, we have developed a new scanning optical microscopy (SOM) imaging method which directly localizes both open and shorted interconnections of ICs from the front and backside of the Si substrate. The method uses a scanned infrared laser source to produce localized thermal gradients in the IC interconnections. The effects of the thermal gradients on IC power consumption are detected by monitoring the voltage fluctuations of the IC power supply voltage $\left(V_{D D}\right)$ with the IC biased using a constant current power supply. Open conductors are detected using the Seebeck Effect to change the power demands of the IC. Conductor short sites are localized using the resistance change described by OBIRCH and the enhanced detection sensitivity provided by constant current biasing. The Thermally-Induced Voltage Alteration (TIVA) images can localize short sites in a single, entire die field of view image.

Since the initial development of TIVA and SEI, efforts have centered on improving the sensitivity of the techniques. Recent system modifications have greatly increased the defect detection sensitivity of both techniques, resulting in faster failure localization and diagnosis.

The laser beam and defect interaction physics used to generate Seebeck Effect Imaging (SEI) and TIVA images are described. The data acquisition system, protocols for image collection, and areas for system improvement are also discussed. Several examples demonstrating the utility of thermal gradient effects for localizing open and shorted interconnections from the front side and backside of ICs are presented. Subsequent system improvements and imaging examples illustrating the utility of the enhanced TIVA and SEI techniques are described. Modeling results 
showing the thermal distribution and response of static and dynamic laser heating are also discussed.

\section{Imaging System}

A block diagram of the SOM system used for SEI and TIVA imaging is shown in Fig. 1. The SOM is a Zeiss Laser Scan Microscope. A $1340 \mathrm{~nm}, \mathrm{Nd}: \mathrm{YVO}_{4}$ laser illumination source and a Ge diode photo-detector produce reflected light images. A Keithley 238 source measurement unit provides electrical stimulus to the IC and an Ithaco $1201 \mathrm{~B}$ voltage amplifier detects voltage variations in $V_{D D}$. The amplifier is operated in the ac-coupled mode so that only the variations in $V_{D D}$ are used to make the images.

The SOM and system components are similar to those described for backside LIVA imaging [5], with the important difference being a change in laser wavelength to $1340 \mathrm{~nm}$. The photocurrent effects from electron-hole pair generation by the $1064 \mathrm{~nm}$ laser used in LIVA produce signals 1 to 2 orders of magnitude larger than the SEI and TIVA signals and must be avoided. The 1340 $\mathrm{nm}$ wavelength illumination source eliminates the LIVA signal because the energy of the photons is below that of the $\mathrm{Si}$ indirect band-gap, and thus no electron-hole pairs are produced. In practice, when using amplification gains of 5000 or more on the Ithaco we observed some LIVA signals. The specifications for our laser indicate that $<1 \%$ of the laser power may be at a $1047 \mathrm{~nm}$ wavelength. To eliminate the unwanted LIVA signals, a $4^{\text {th }}$ order, long wavelength pass filter was placed in the beam path as shown.

Typical image scan times of 4 to 8 seconds per frame were used. The relatively long scan times were required to compensate for the loss in amplifier bandwidth at high gains. Line averaging of 8 to 16 lines was also used to improve the image signal to noise ratio. For particularly weak signals, image averaging of 4 to 16 frames was also performed as indicated in the text. System improvements that would increase SEI and TIVA signal strength are described in the Future Research section.

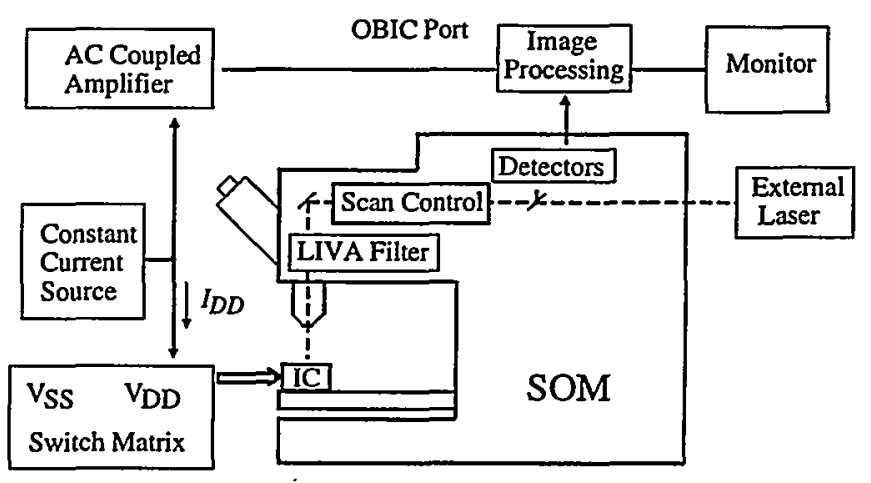

Fig. 1. SOM system used for SEI and TIVA. 


\section{Seebeck Effect Imaging}

Thermal gradients in conductors generate electrical potential gradients with typical values on the order of $\mu \mathrm{V} / \mathrm{K}$ [6]. This is known as thermoelectric power or the Seebeck Effect and refers to the work of Thomas Johann Seebeck (1770-1831)[7]. The most common application of thermoelectric power is the thermocouple, which uses the difference in thermoelectric voltages of two different metals to measure temperature (Fig. 2a.). In a single element the main driving force that produces a voltage gradient is the thermal motion of electrons away from high temperature areas towards cooler regions (Fig. 2b.). For IC analysis, the effect has been demonstrated as a means to localize voiding in metal test patterns [8]. If an IC conductor is electrically intact and has no shorts, the potential gradient produced by localized heating is readily compensated for by the transistor or power bus electrically driving the conductor and essentially no signal is produced. However, if the conductor is electrically isolated from a driving transistor or power bus, the Seebeck Effect will change the potential of the conductor. This change in conductor potential will change the bias condition of transistors whose gates are connected to the electrically open conductor, changing the transistors' operating condition and power dissipation. An image of the changing IC power demands displays the location of electrically floating conductors.

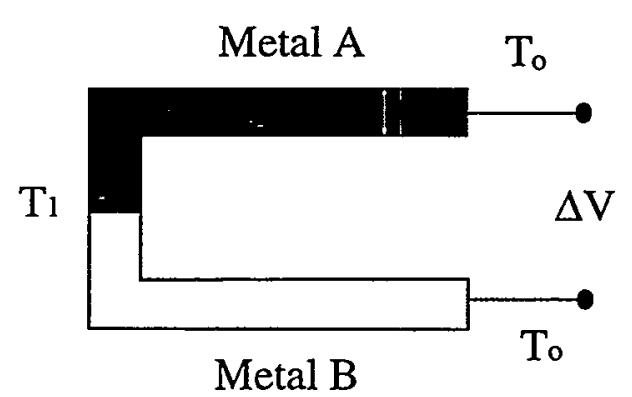

a)

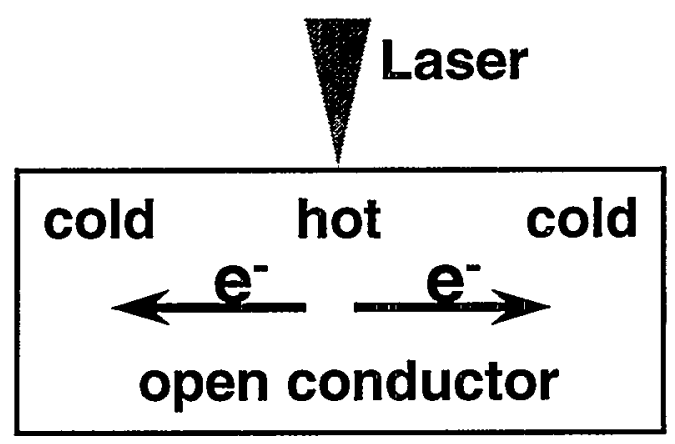

b)

Fig. 2. a) Elementary thermocouple and b) the motion of electrons due to a thermal gradient.

The results of thermal modeling at Sandia showed that a focused $1340 \mathrm{~nm}$ laser will produce about a $1{ }^{\circ} \mathrm{C} / \mathrm{mW}$ temperature change in a Si substrate. At present a $120 \mathrm{~mW}$, single mode, polarized laser is used for thermal stimulus. The transmission efficiency of our SOM (about 3\% to $25 \%$ depending upon the objective lens) indicates that thermal gradients on the order of $4{ }^{\circ} \mathrm{C}$ to $30^{\circ} \mathrm{C}$ can be produced, creating potential variations in the 1's to $10^{\prime} \mathrm{s}$ of $\mu \mathrm{V}$. Essential to SEI localization of open interconnections is detection of the small changes in transistor gate voltages with thermal gradients. Detection of the small variation in transistor bias condition is accomplished by using constant current biasing of the IC. The Light- and Charge- Induced Voltage Alteration (LIVA and CIVA) imaging techniques $[3,9,10]$ and transient voltage $\left(v_{D D T}\right)$ test method [11] have shown that monitoring the supply voltage to the IC with constant current biasing conditions provides an extremely sensitive method for detection of subtle changes in the IC power demand. Fig. 3 displays a comparison of IC current increase using constant voltage biasing to IC voltage decrease using constant current biasing for a CMOS ASIC. For example, a power demand change that would produce a $35 \mathrm{nA}$ change in $I_{D D Q}$ at a constant voltage will produce a $1 \mathrm{~V}$ change in $V_{D D Q}$ at constant current. For very low power CMOS devices, IC power 
changes corresponding to a supply current increase of $100 \mathrm{pA}$ for a constant supply voltage have been shown to produce a $2 \mathrm{~V}$ supply voltage decrease for constant supply current biasing. This sensitivity is critical in detecting the potential gradients produced by the Seebeck effect. Typical amplifier gains used for SEI in this work range from 5000 to 10,000 . For our system this indicates $V_{D D}$ changes on the order of $0.1 \mathrm{mV}$. The current changes with constant voltage biasing were too small to be detected with our present system. The $1340 \mathrm{~nm}$ wavelength is suitable for effective Si transmission and conductor heating, will not produce electron-hole pairs (avoids photocurrent effects), and yields reasonable spatial resolution in a reflected light image. Note that changing the voltage of open conductors that do not drive transistor gates (for example an open power bus) may not change the power demand of the IC and will therefore not be detected using SEI.

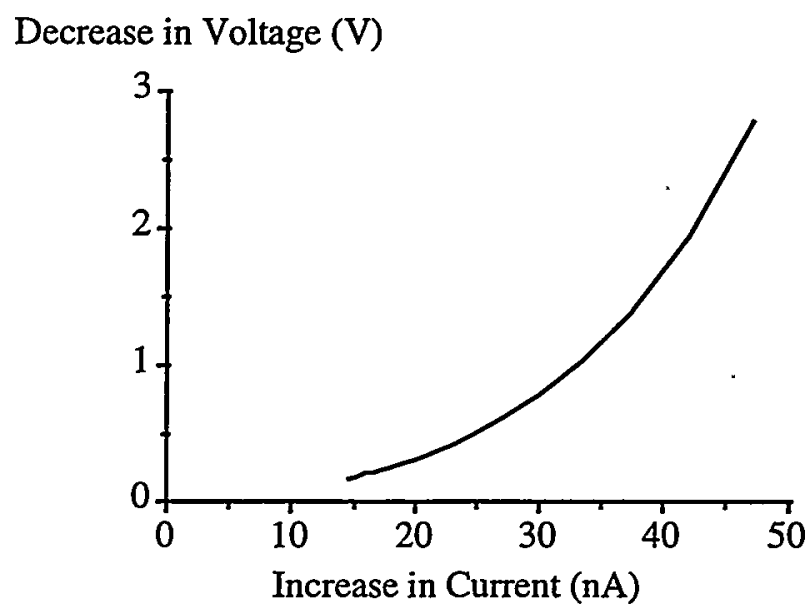

Fig. 3. Sensitivity comparison of constant voltage and constant current biasing under the same stimulus.

The SEI signal is produced by heating the open conductor which occurs even if the scanned thermal probe is larger than the conductor. The spatial resolution of SEI will be limited by the $1340 \mathrm{~nm}$ wavelength, however the detection sensitivity of SEI image contrast depends on the conductor being examined and not on the probe size. Therefore, the image of the open interconnection may appear larger than the actual conductor, but will effectively localize the open signal path.

\section{TIVA Imaging}

Localized thermal gradients can also detect shorted IC conductors. Shorted conductors cause increased IC power consumption when the shorted conductors are at different electrical potentials, i.e. a short between $V_{D D}$ and $V_{S S}$. The power consumption will depend upon the resistance of the short site and its location in the circuit. A low resistance $V_{D D}$ to $V_{S S}$ power bus short may dissipate more power than two shorted signal lines. As a laser is scanned over an IC with a short circuit, laser heating changes the resistance of the short when it is illuminated, changing the IC power demand. The resistance change can be expressed as [12]: 


$$
\rho=\rho_{o}\left(1+\alpha\left(T-T_{0}\right)\right)
$$

where $\rho$ is the resistivity, $\rho_{0}$ is the resistivity at $T_{0}, \alpha$ is the temperature coefficient of resistivity, $\mathrm{T}$ is the temperature and $\mathrm{T}_{\mathrm{o}}$ is the reference temperature.

It has been found that thermally-induced power changes are usually greater for shorted signal lines than power busses. This results from signal line voltage fluctuations altering transistor gate voltages, producing the same amplification effect observed in CIVA and SEI. This resistance change with localized heating is the basis for the OBIRCH technique [5]. For OBIRCH, the change in IC power consumption is detected by an IC current change with constant voltage bias, yielding limited detection sensitivity [5]. The constant supply current biasing approach achieves greater sensitivity and a TIVA image is produced localizing the short.

Therefore, using the same IC biasing and laser stimulus, both open and shorted conductors can be localized from the front and backside of an IC.

\section{Imaging Examples}

\subsection{Seebeck Effect Imaging}

Fig. 4(a) is an SEI image from the front side of an IC. The IC is a radiation-hardened, $4 \mu \mathrm{m}$ metal, $3 \mu \mathrm{m}$ polysilicon, CMOS version of an Intel 8085 microcontroller manufactured at Sandia using a single level metal process. The open site was produced by laser ablation. Fig. 4(b) is a reflected light image of the same field of view for registration. Note that the entire die is examined in a single image. The floating conductor path and the transistors controlled by the conductor are visible in Fig. 4(a). The transistor diffusions appear bright compared to the open conductor contrast. We believe the bright signals result from the diffusion to well thermopower difference, changing the voltage of gates downstream of the floating gate transistors when heated. In effect, the junction region is acting like a small thermocouple.

Fig. 5(a) shows the same device in Fig. 4 at higher magnification. The laser ablation site is indicated by an arrow. The SEI images are reminiscent of CIVA images showing open conductors but with reduced signal strength.

An SEI section of the floating conductor in Fig. 4 is displayed at higher magnification in Fig. 6(a). Contrast enhancement and superposition with the reflected light image localize the open conductor.

Somewhat surprising is the fact that we do not see a contrast change in metal-polysilicon interconnections on this older Sandia technology. (In later examples we do observe contrast in the electrically floating metal-polysilicon interconnections.) The metal conductors in this older technology were processed without $\mathrm{Cu}$ doping of the $\mathrm{Al}$. The difference in processing appears to have resulted in a much smaller thermopower difference than seen in other interconnections below. 


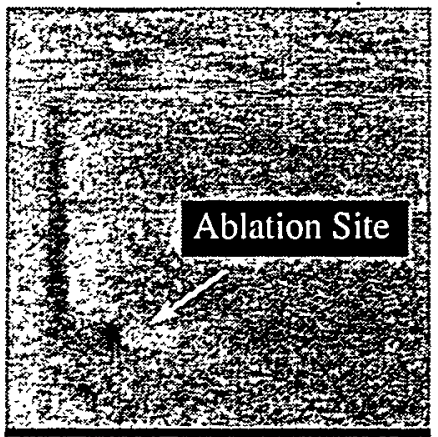

(a)

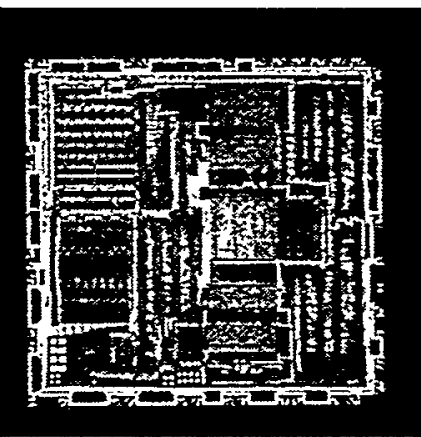

(b)

Fig 4. (a) Front side SEI image of a floating conductor. (b) Reflected light image with the same field of view for reference.

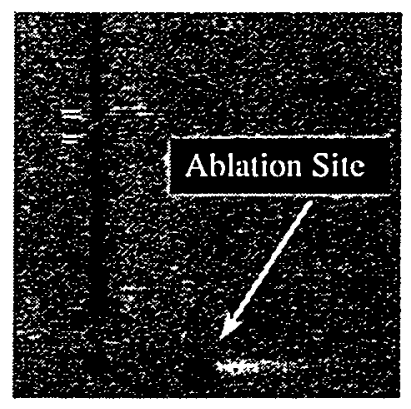

(a)

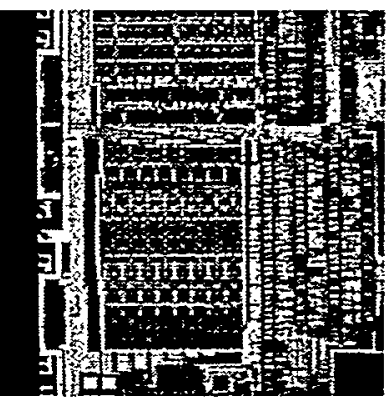

(b)

Fig 5. (a) Higher magnification front side SEI image of the floating conductor in Fig. 4. (b) Reflected light image of the same field of view for reference.

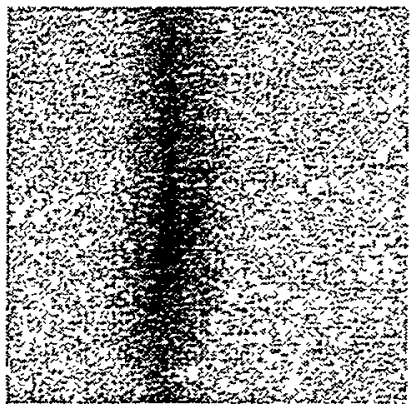

(a)

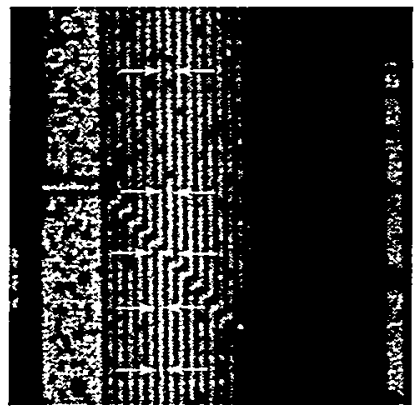

(b)

Fig. 6. (a) Front side SEI image section of a floating conductor. (b) Superposition of the image in Fig. 6(a) and a reflected light image of the same field of view.

Fig. 7 is another front side SEI example of a open conductor on a radiation-hardened CMOS version of an Intel 80C51 microcontroller manufactured at Sandia using a two-level metal, 1.25 $\mu \mathrm{m}$ process. The open was created with a focused ion beam (FIB). Fig. 7(a) shows the open site with the long wavelength pass filter in place. Fig. 8(a) displays the same open site with the filter removed. The blurred contrast visible in Fig. 8(a) is a LIVA signal produced from the $1047 \mathrm{~nm}$ 
component of the laser. The signal appears blurred because the focus is optimized for the 1340 $\mathrm{nm}$ wavelength. The filter was left in place for subsequent images to eliminate the LIVA signals.

A higher magnification view of the open conductor shown in Figs. 7 and 8 is shown in Fig. 9(a). The arrow indicates the FIB open site. Note that not only is the open conductor visible, but a weaker and somewhat diffuse signal can also be seen from the conductor and gates beneath the upper metal-2 power bus. The contrast from the lower structures indicates that the thermal gradient on the surface of the metal-2 has propagated through the metal and interlevel dielectric to the metal-1 and polysilicon conductors underneath. This thermal conduction is consistent with the modeling work shown recently by Ferrier [13] for subsurface, heat producing defects.

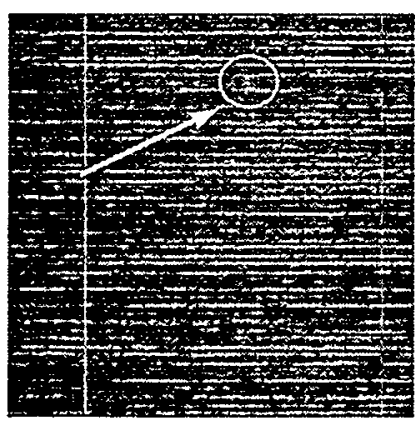

(a)

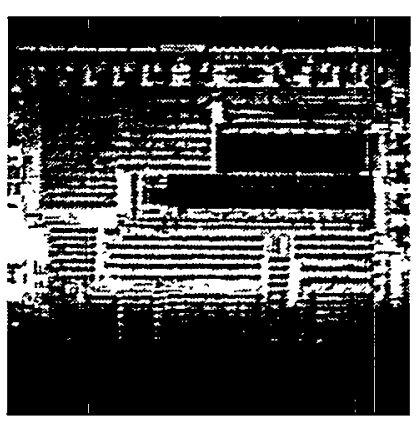

(b)

Fig. 7. (a) Front side SEI image and (b) reflected light image of an open conductor on a microcontroller.

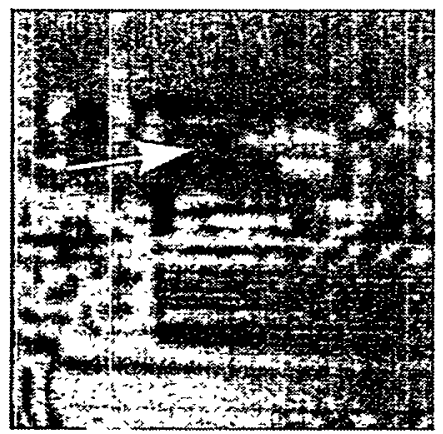

(a)

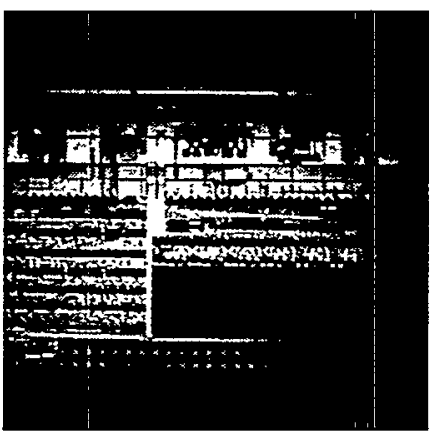

(b)

Fig. 8. (a) SEI and (b) reflected light image pair similar to Fig 7 with the long wavelength pass filter removed. The blurred contrasts in Fig. 8(a) are from LIVA signals. The SEI signal is indicated by the arrow.

The microcontroller in Fig. 9 could be clocked to a different state where a much stronger contrast was observed. This contrast (Fig. 10) appears to be originating from under the power bus but could not be localized further from the front side of the IC. 


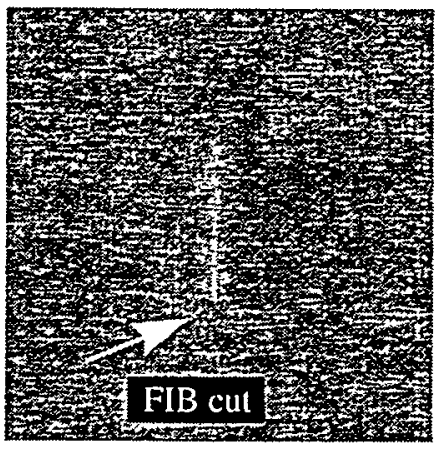

(a)

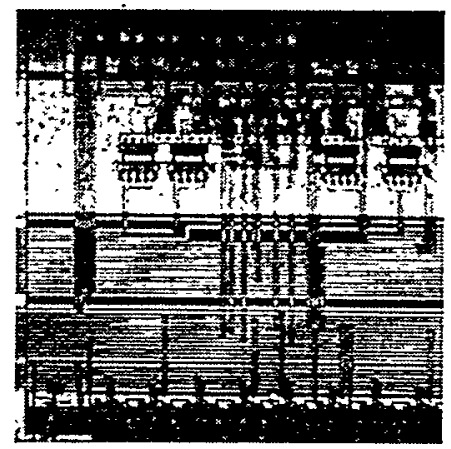

(b)

Fig. 9. Higher magnification image of the microcontroller using (a) SEI and (b) reflected light imaging of the open conductor in Fig. 7.

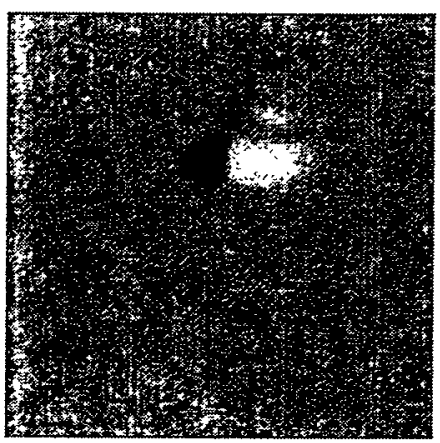

(a)

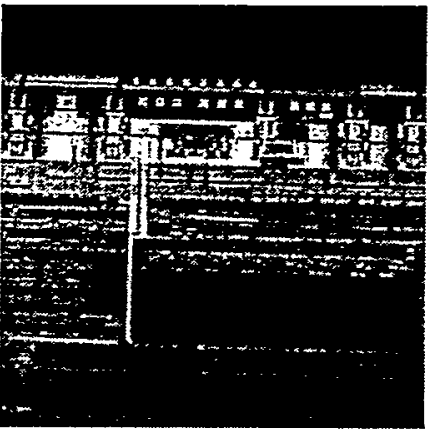

(b)

Fig. 10. (a) Strong SEI contrast signal produced by clocking the microcontroller and (b) reflected light image.

Backside SEI was performed using the same $1340 \mathrm{~nm}$ wavelength laser for stimulus. The signal strength is reduced for backside examination because of absorption by doped Si. Fig. 11 shows the percent transmission as a function of wavelength and $p$-doping concentration for $625 \mu \mathrm{m}$ of $\mathrm{Si}$ [14]. Heavier doping will increase the absorption. Thinning of the sample improves signal strength. The percent transmission of infrared wavelengths is expressed by [14]:

$$
\% \mathrm{~T}=\frac{(1-\mathrm{r})^{2} \mathrm{e}^{-\alpha d}}{1-\mathrm{r}^{2} \mathrm{e}^{-2 \alpha d}} \times 100 \%
$$

where $\% \mathrm{~T}$ is the percent transmission, $\mathrm{r}$ is the reflection coefficient, $\alpha$ is the absorption coefficient, and $\mathrm{d}$ is the Si thickness. Decreasing the thickness can dramatically increase the \%T and therefore the SEI signal. No thinning, beyond backside exposure, was performed on the samples used in this work. 


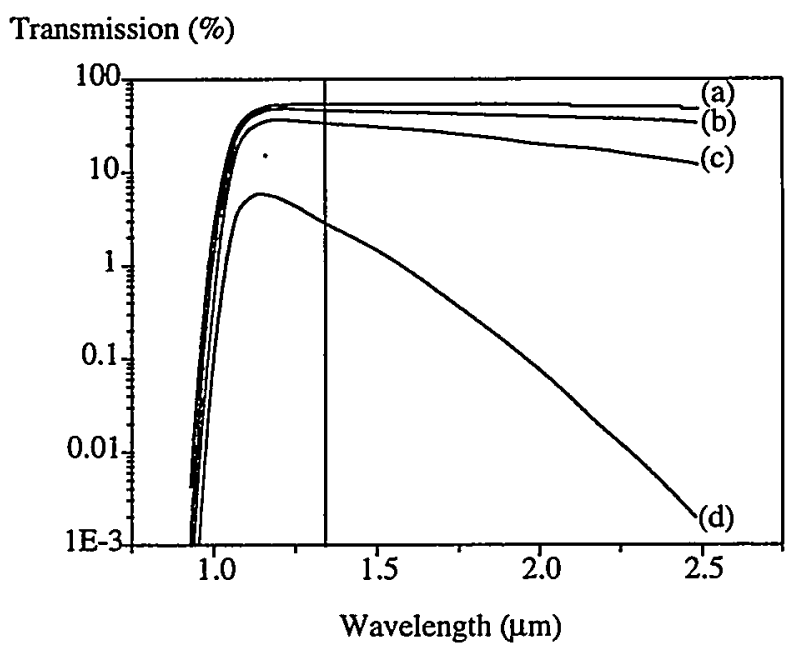

Fig. 11. Percent transmission of light through $625 \mu \mathrm{m}$ of $p$-doped Si having doping concentrations of (a) $1.5 \times 10^{16}$, (b) $3.3 \times 10^{17}$, (c) $1.2 \times 10^{18}$, and (d) $7.3 \times 10^{18} \mathrm{~cm}^{-3}$. The 1340 $\mathrm{nm}$ wavelength is marked by a vertical line.

Fig 12 is a backside SEI imaging example of a floating conductor. The floating conductor was produced by laser ablation. The IC is a low power CMOS ASIC manufactured at Sandia in a 4 $\mu \mathrm{m}$ metal, $3 \mu \mathrm{m}$ polysilicon process. The sample was potted in epoxy and planar lapped to expose the back of the die. The arrow indicates the laser ablation site. The floating conductor path from the open site to the driven transistor gate can be seen. Note that the CMOS logic gates can be seen as a weak background signal (Fig. 12(a)). These are not produced by a LIVA effect, but are probably produced by directly heating the diffusions using the $1340 \mathrm{~nm}$ laser. This imaging of the logic states was observed only on this low power ASIC. We believe that the signal originates from the thermocouple effect seen earlier and exists on all samples examined, but is too weak to be seen on any of the other devices examined other than this low power IC.

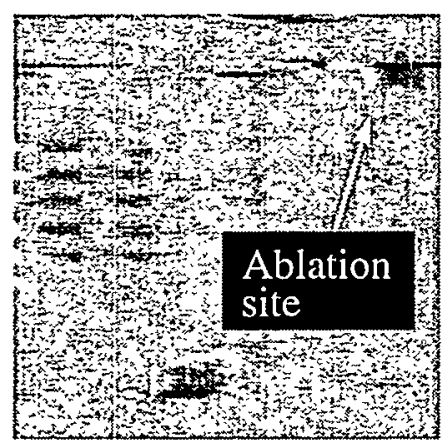

(a)

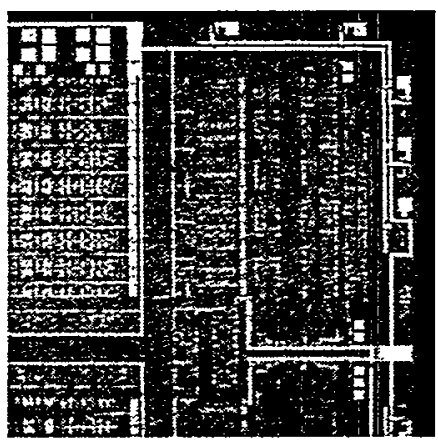

(b)

Fig. 12. (a) Backside SEI imaging example of a floating conductor. (b) Reflected light micrograph of the same field of view.

A higher magnification backside view of the laser ablation site is shown in Fig. 13. Fig. 13(a) is an average of 4,8 second per frame scans, each scan having 16 line averaging. The open site is indicated by the arrow. In addition to the floating conductor, there are two features of note in 
Fig. 13(a). First, a "halo" of image contrast is present about the laser ablation site. In the reflected light image (Fig. 13(b)) we see that this area is unpopulated Si. The lateral thermal conduction efficiency of $\mathrm{Si}$ is relatively high. As areas of the $\mathrm{Si}$ surface are heated by the focused laser beam with no intervening structures to absorb the heat, the floating conductor is heated and the Seebeck Effect occurs. The resulting effect is a less localized SEI image due to thermal spreading in the Si. A second feature of Fig. 13(a) is the strong contrast in the region where the conductor goes over the field oxide and $p$-well of logic gates. We believe that the these features reduce thermal loss through the $\mathrm{Si}$ and increase the thermal gradient along the conductor. Contrast changes at the polysilicon-metal interconnections are not visible in Fig. 13(a). The IC was produced in the process described earlier that does not use $\mathrm{Cu}$ doping of the Al.

Fig. 14(a) is a backside SEI example of the open conductor shown in Figs. 7 and 9. Note that even with a relatively poor polish (Fig 14(b)) the open site can be identified. At higher magnification the open FIB site and floating conductor can be seen (Fig. 15(a)). Fig. 15(a) is an average of 16, 8 second per frame scans, each scan having 16 line averaging. Relatively strong contrasts were produced by the metal-1 ( $\mathrm{Cu}$ doped) to polysilicon interconnections. Observe that the contrast changes from dark, to bright, to dark at metal-poly, poly-metal, and metal-poly interconnections respectively. We believe this is due to a thermocouple effect between the polysilicon and metal conductors.

The microcontroller could be clocked to the same state shown in Fig. 10 and imaged from the backside. Fig. 16 is a backside SEI image of this logic state. The signal strength of the contrast site was approximately 2 orders of magnitude greater that the signals in Figs. 14 and 15. The $p$ channel transistor generating the contrast can be identified. The signal is produced as the downstream gates driven by the floating transistor have their voltage changed. We believe that the signal is stronger because of a thermocouple effect between the $p$-diffusion and $n$-well of the floating gate transistor.

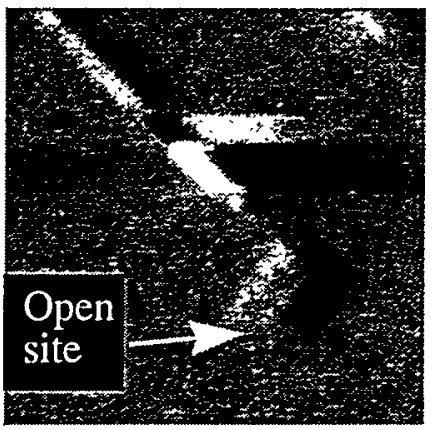

(a)

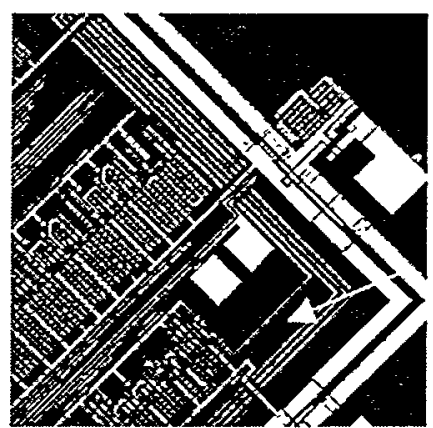

(b)

Fig 13. (a) Higher magnification backside SEI and (b) reflected light image pair of the defect shown in Fig. 12. 


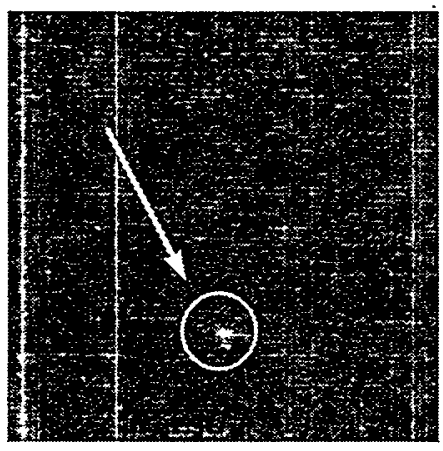

(a)

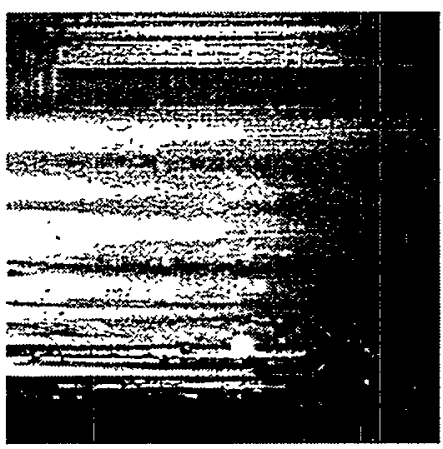

(b)

Fig. 14. (a) Backside SEI and (b) reflected light images of the open conductor show in Figs. 7 and 9.

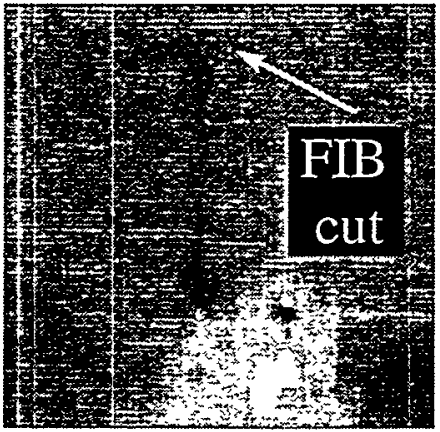

(a)

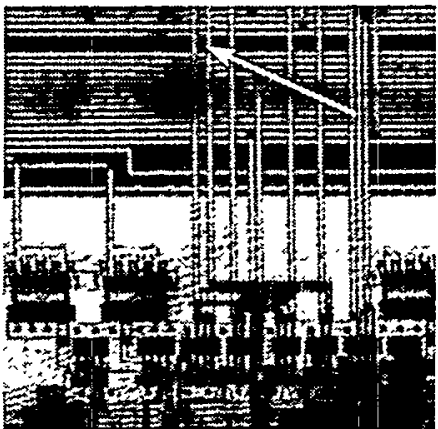

(b)

Fig. 15. (a) Higher magnification backside SEI and (b) reflected light images of the open conductor shown in Fig. 14.

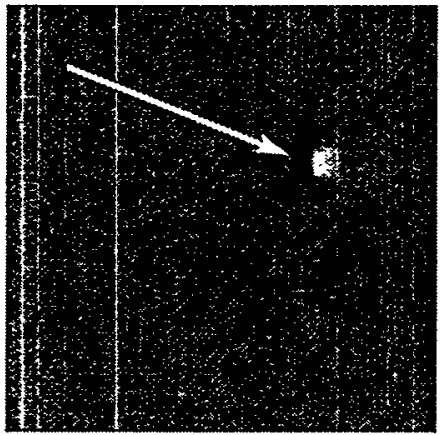

(a)

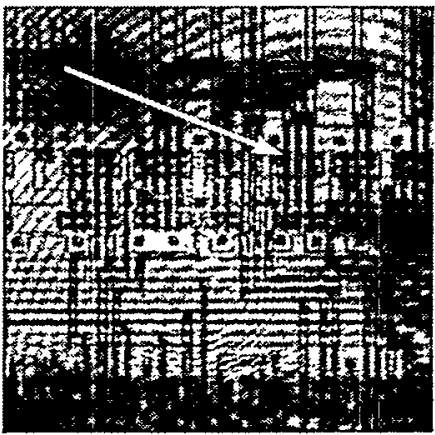

(b)

Fig. 16. (a) Strong backside SEI image contrast from the microcontroller of Fig. 14 clocked to a different logic state. (b) Reflected light image of the same field of view. 


\subsection{TIVA Imaging}

Fig. 17 displays a front side TIVA image example of a FIB produced short on a 3-level metal, 0.5 micron, 256K SRAM. Fig. 18 is a higher magnification view of the memory. The FIB short is the dark, rectangular contrast seen in Fig 18(b). Fluorescent Microthermal Imaging (FMI) [15] identified the bright contrast indicated by the arrow as a heat producing source, but not the additional contrast sites seen in the TIVA image. Subsequent LIVA analysis of the IC with a visible, $5 \mathrm{~mW}, 543 \mathrm{~nm}$ wavelength laser identified the additional contrast sites seen in the TIVA image but not the short site found in the TIVA and FMI images. The spurious TIVA and LIVA contrast sites are transistor diffusions connected to the shorted conductor and are therefore not connected directly to $V_{D D}$ or $V_{S S}$. We believe that the thermocouple effect seen before changes the voltage of these diffusions and the transistor gates they drive. Similarly, the photocurrents induced by LIVA will have the same effect and produce the similar LIVA contrast sites observed. The heating effects of the $5 \mathrm{~mW}, 543 \mathrm{~nm}$ laser are not significant compared to its electron-hole pair generation.

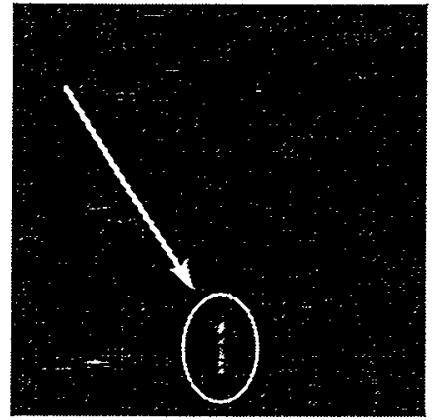

(a)

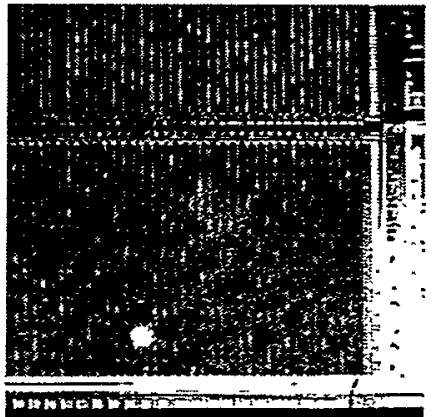

(b)

Fig. 17. (a) Front side TIVA image of a FIB short on a 256K SRAM. (b) Reflected light image of the same field of view. The bright spot in the bottom of (b) is caused by internal reflection in the SOM.

A front side TIVA image of an entire, 3-level metal, $0.5 \mu \mathrm{m}, 1 \mathrm{MB}$ SRAM is shown in Fig. 19. The TIVA short site was identified as a stainless steel particle shorting two signal lines. Fig 20 is a higher magnification view of the short site. The particle causing the short and a small section of the shorted conductors can been seen in Fig 20(a). The particle is visible in Fig 20(b).

Fig. 21 is a front side TIVA image of a stainless steel particle short between two signal lines on another 1 MB SRAM. The particle is clearly seen in Fig 21(b), but the spatial resolution is not sufficient to see the individual conductors. The surface of this memory was sealed with epoxy and polished for backside TIVA analysis. 


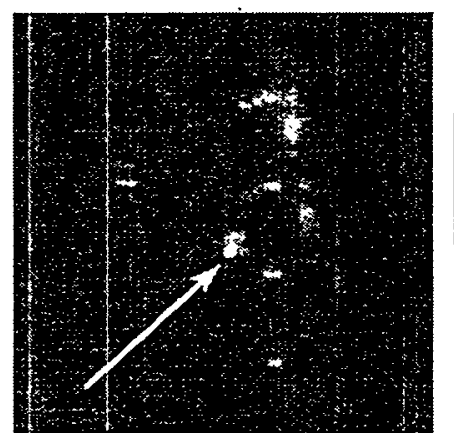

(a)

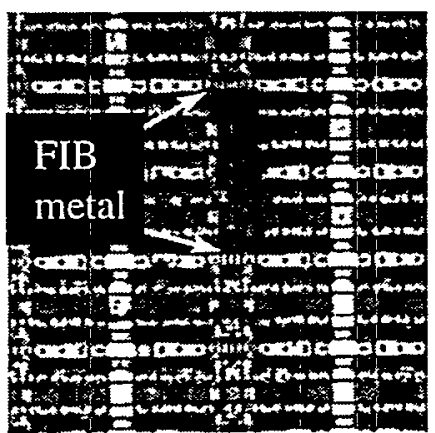

(b)

Fig. 18. Higher magnification front side TIVA and reflected light images of the FIB short site in Fig. 17. The arrow indicates the hot spot found using FMI. The rectangular FIB deposited metal can be seen in Fig 18(b).

The backside TIVA image acquired is shown in Fig. 22. The arrow highlights a very small TIVA contrast site. Fig. 22a was contrast enhanced to make the small contrast site visible. The SRAM was biased with $4 \mathrm{~mA}$ and reached a voltage of $4.6 \mathrm{~V}$. The voltage changes from the TIVA contrast site were about $0.3 \mathrm{mV}$. No supply current changes were observed using a $5 \mathrm{~V}$ constant voltage bias arrangement. Fig. 23 is a higher magnification backside TIVA image of the short site. Note that while the short site is clearly visible in the TIVA image, the particle, now under the metal conductors, cannot be seen in the reflected light micrograph (Fig. 23(b)).

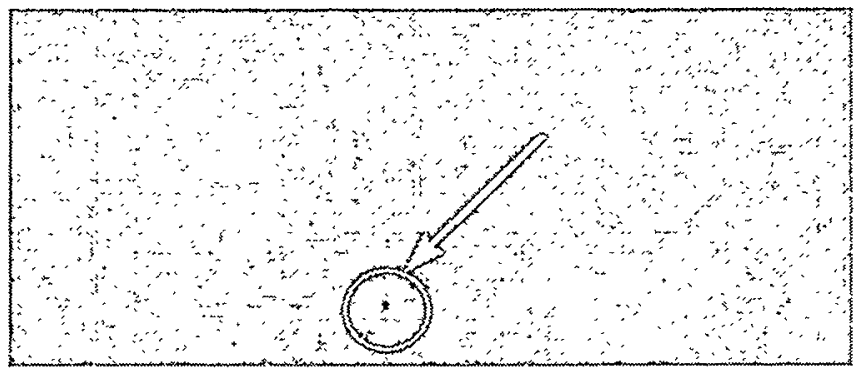

(a)

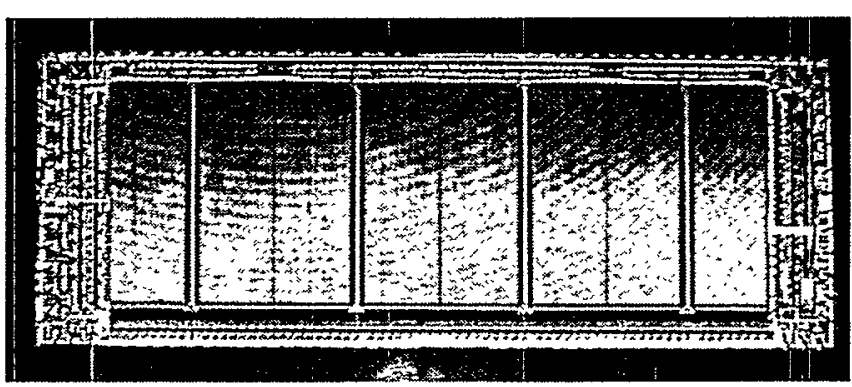

(b)

Fig. 19. (a) Front side TIVA image of a 1 MB SRAM. The arrow indicates the short site. (b) Reflected light image for registration. 


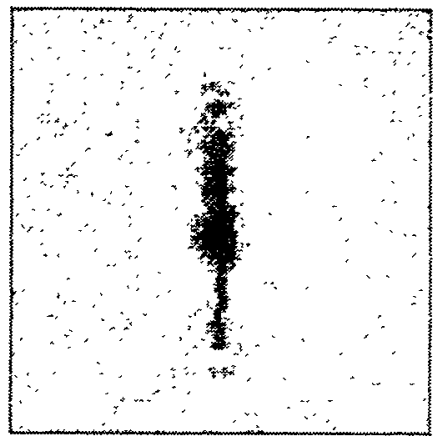

(a)

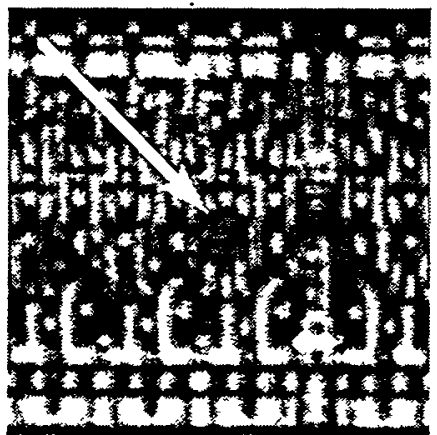

(b)

Fig. 20. Higher magnification front side TIVA and reflected light image pair of the short site in Fig. 19. The shorting particle can be seen in Fig. 20(b). The blurred appearance of Fig. 20 (b) demonstrates the spatial resolution limitations of imaging with the $1340 \mathrm{~nm}$ wavelength laser.

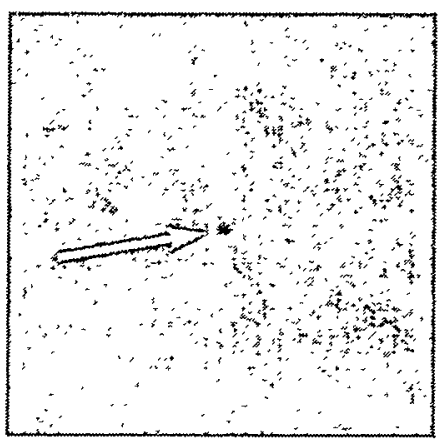

(a)
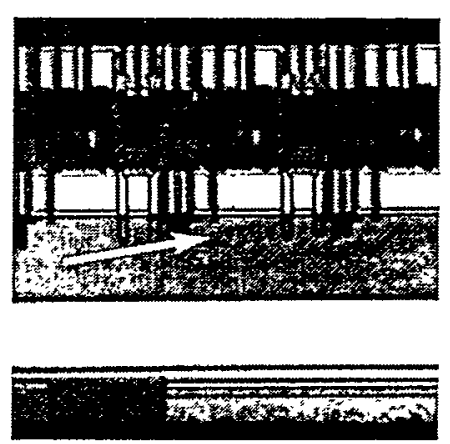

(b)

Fig. 21. Front side TIVA and reflected light image pair showing a particle short on the $1 \mathrm{MB}$ SRAM.

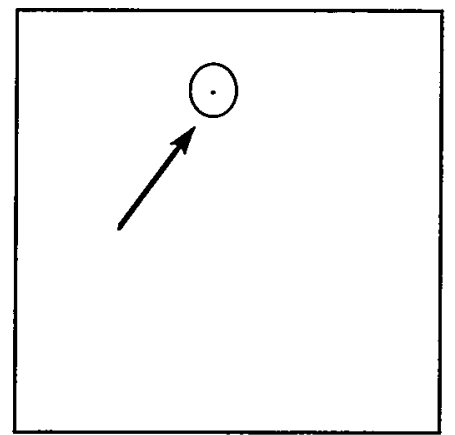

(a)

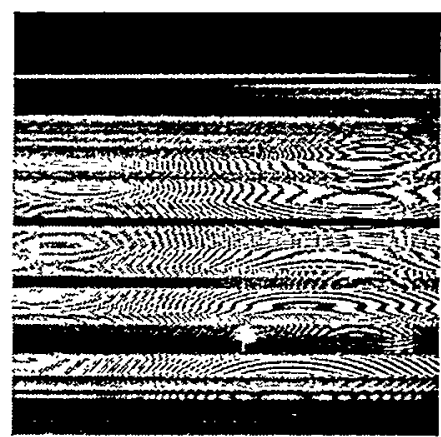

(b)

Fig. 22. (a) Backside TIVA image locating the short on the SRAM from Fig 21. (b) Reflected light image for registration. 


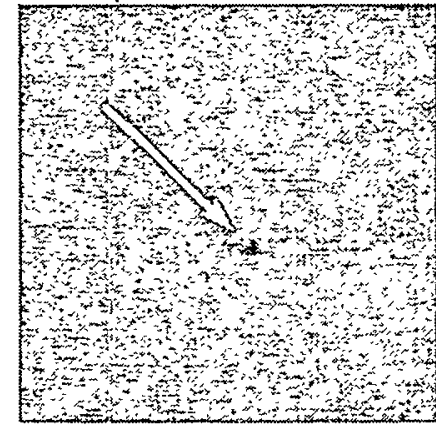

(a)

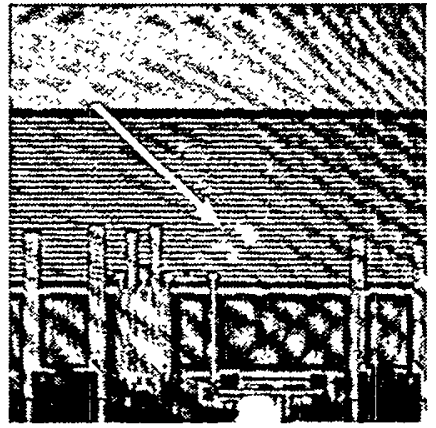

(b)

Fig 23. Higher magnification backside TIVA and reflected light image pair of the short site from Fig 22. Note that the particle cannot be seen from the backside in Fig. 23(b).

\section{System Improvements and Signal Increases}

Several improvements to the TIVA/SEI system described above have produced enhanced sensitivity in detecting open and shorted interconnections on ICs. The improved sensitivity is due to an increase in the available laser power (heat) delivered to the IC and to the non-linear thermal response of the IC defect.

The increase in power comes from two sources. First, heating has been increased by simply using a higher power laser. The earlier TIVA/SEI work was performed using a $120 \mathrm{~mW}$ laser. Measurements are now done using a $430 \mathrm{~mW}$ laser. Second, a half-wave plate has been inserted into the beam path where the laser enters the scanning laser microscope. By properly orienting the half-wave plate polarization reflection losses are minimized. The half-wave plate increases the transmission efficiency of the microscope by approximately $30-50 \%$. The resulting increase in power from the new laser and half-wave plate is approximately $5 \mathrm{X}$.

The non-linear thermal response of defects greatly amplifies the benefits of increased laser power. While the exact response of the IC will depend upon the location and nature of the defect, a front side short example from a microprocessor demonstrates the non-linearity. Fig. 24 shows the change in supply voltage under constant current biasing with different laser intensities on the short. The amplitude of the signal roughly quadruples as the laser intensity doubles. Our earlier work with the lower power laser and without the half-wave plate produced much weaker TIVA/SEI signals, the equivalent of using a $60 \mathrm{~mW}$ laser in Fig 24.

\section{Initial Modeling of Thermal Properties}

Thermal modeling was performed to determine the temperature distributions obtainable with static and pulsed laser illumination. Details of the test chip geometry were simulated with a 3D numerical model. A commercial software product, TAS, was used to solve the heat transfer problem [16]. This code is a comprehensive tool that uses a finite difference solver that was tested against numerous benchmark problems for accuracy and is used extensively in electronic packaging analysis. In this model, the material properties for each of the materials were included. 


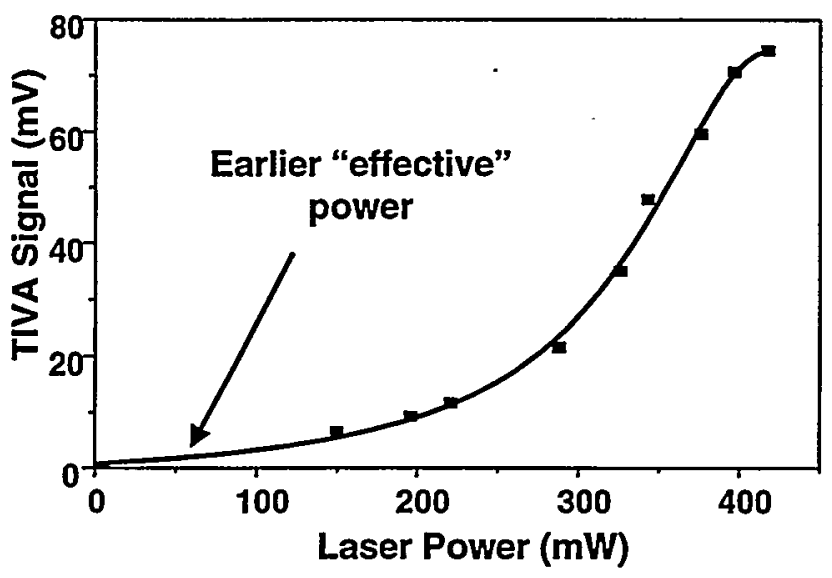

Fig. 24. Non-linear response of defects to laser intensity.

The initial modeling work assumed a $120 \mathrm{~mW}$ incident laser focused to the front of the $\mathrm{Si}$ through the backside of a $50 \mu \mathrm{m}$ thick sample. The temperature rise in the model was found to scale linearly with incident laser power.

The temperature variations with a 50\% duty cycle pulsed beam at $1 \mathrm{MHz}$ and $100 \mathrm{MHz}$ are show in Figs. 25 and 26. Note that there is a fairly large temperature change even at $100 \mathrm{MHz}$.

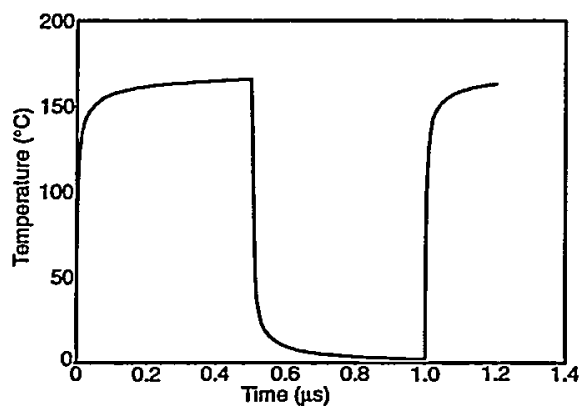

Fig. 25. Surface temperatures at the center of the laser heated spot for the base calculation case with a $1 \mathrm{MHz}$ square wave modulated power input to the die.

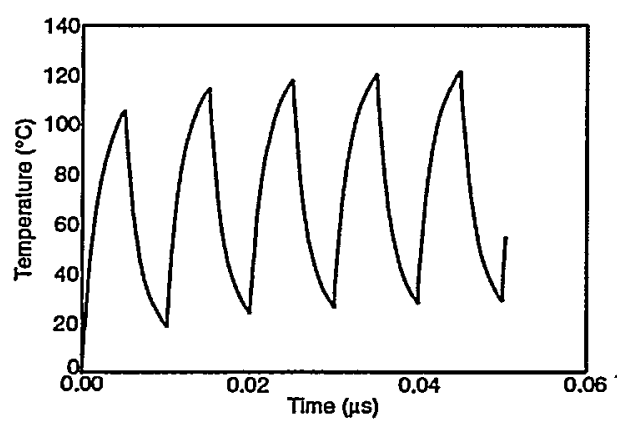

Fig. 26. Surface temperatures at the center of the laser heated spot with a $100 \mathrm{MHz}$ square wave intensity modulation. 


\section{Imaging Results with the Improved System}

Several examples are presented to demonstrate the improved system detection sensitivity. Fig. 27 shows a FIB-produced open conductor from the backside of a $1.25 \mu \mathrm{m}$ microcontroller. Note the poor polish in the reflected image. The defect was observed earlier, but required about 45 minutes of averaging to obtain a low noise image. The SEI image in Fig. 27 was acquired in 2 minutes with the improved system.

Fig. 28 shows images of an open conductor site on an older ASIC that has significant electron tunneling across the open [17]. To make the conductor "fail", a $10 \mathrm{~Hz}$ clock line was operated during image acquisition. The resulting front side SEI image localizes the open circuited diffusions (bright contrast) and the open conductor site (dark contrast). The Seebeck Effect voltage gradients are compensated along the length of the conductor by the charge tunneling. At the tunneling site itself, the heat induced voltage gradients and changing tunneling efficiency generate the dark contrast seen.

A final front side SEI example involving open conductors is shown in Fig. 29. The dark areas highlighted by arrows are open conductors produced by mechanical damage on this $0.5 \mu \mathrm{m}$ microcontroller. The image was acquired in 2 minutes.

The TIVA image in Fig. 30 localizes the site of a stainless steel particle short on a $0.5 \mu \mathrm{m}$ SRAM from the backside. The short was visible in earlier work with a 16-minute acquisition time. With the system improvements the acquisition time has been reduced to 2 minutes. Moreover, the two shorted conductors that were not seen in earlier analyses are now clearly visible.

The application of the TIVA technique to a more complicated IC is shown in Figs. 31 and 32. These images were taken through the backside of a 6 level metal, $0.3 \mu \mathrm{m}$ microprocessor. The whole die image (Fig. 31) shows two defects, a damaged input pin on the edge and a central region with suspected capacitor shorts. Fig. 32 is a higher magnification image of the shorted capacitors.
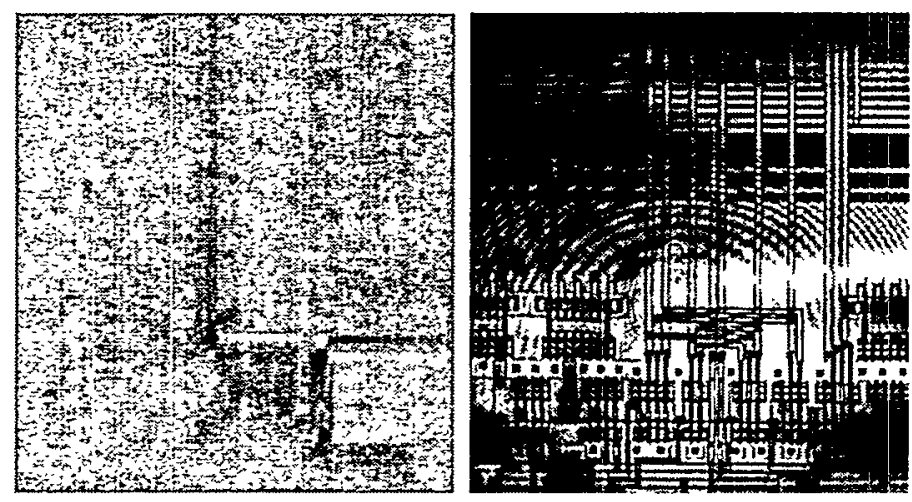

Fig. 27. SEI (left) and reflected light (right) images of an open conductor imaged through the backside of the die. The SEI image was acquired in 2 minutes. On the older system the image required a 45-minute acquisition time to produce similar quality results. 


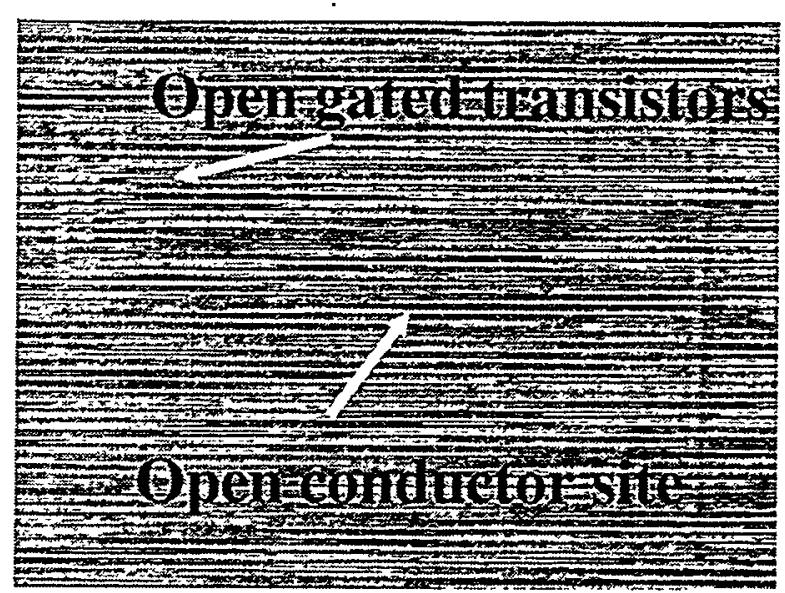

Fig. 28. Front side, whole die image of open circuited diffusions (bright) and open conductor site (dark) localized using SEI.

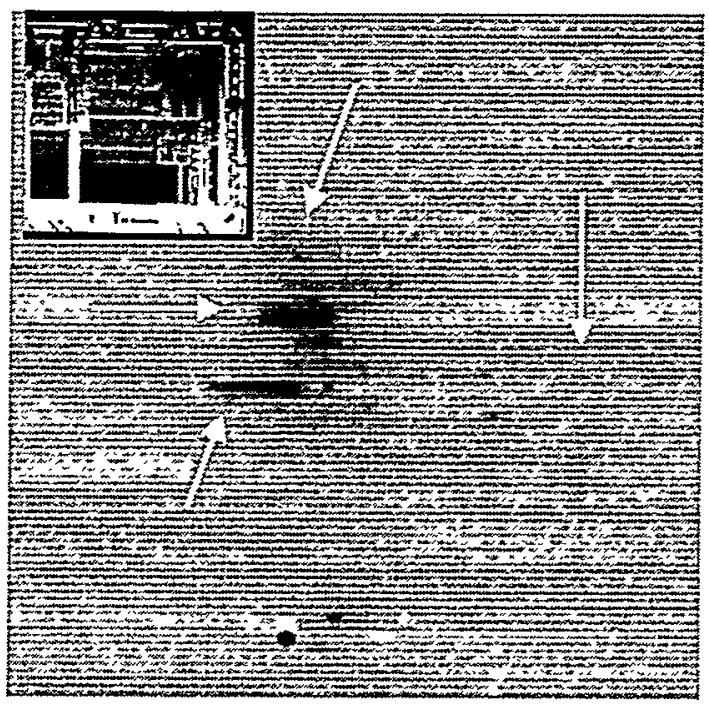

Fig. 29. Front side SEI image showing 4 open conductors. The arrows indicate the open lines. The inset image shows the field of view.
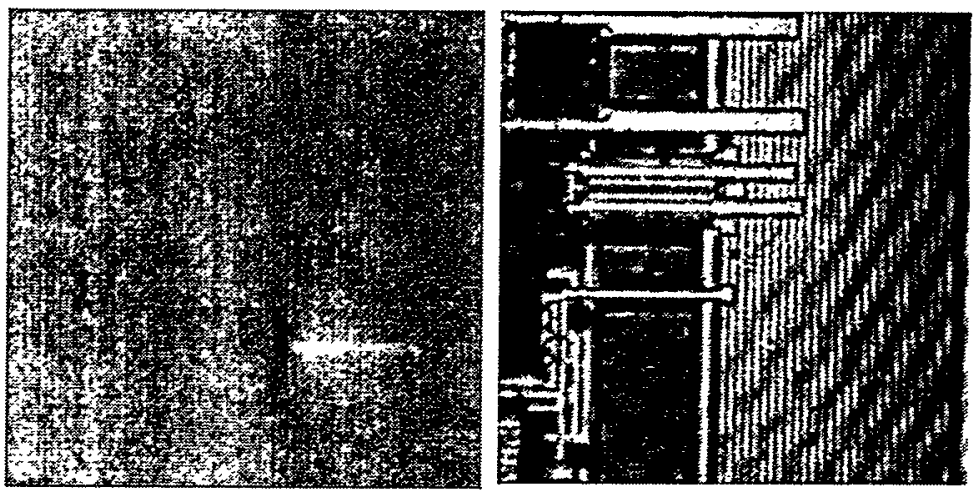

Fig. 30. Backside TIVA (left) and reflected (right) images of a shorted conductor. 

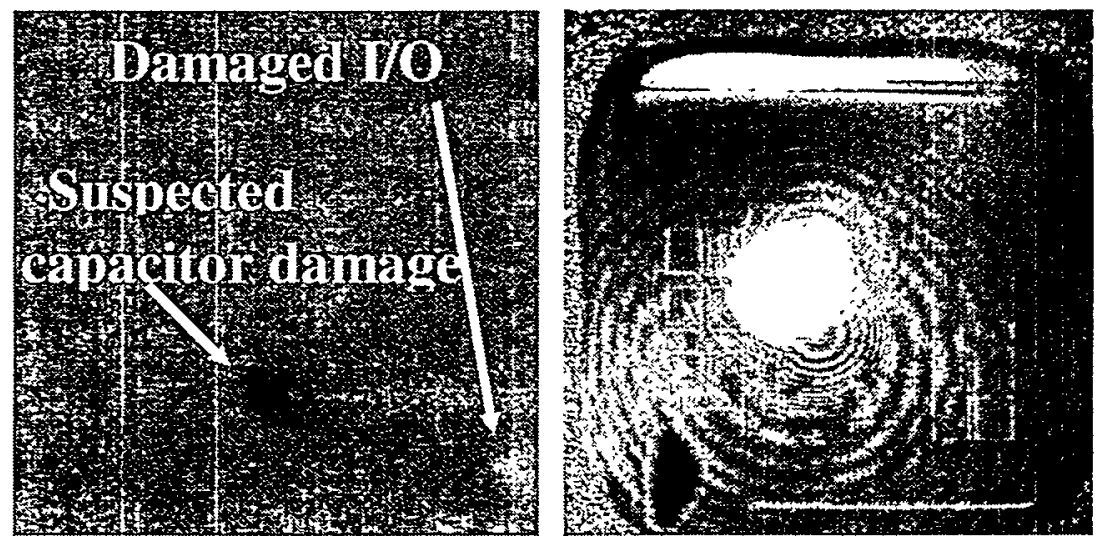

Fig. 31. Backside TIVA (left) and reflected light (right) images of a $0.3 \mu \mathrm{m}$ microprocessor.
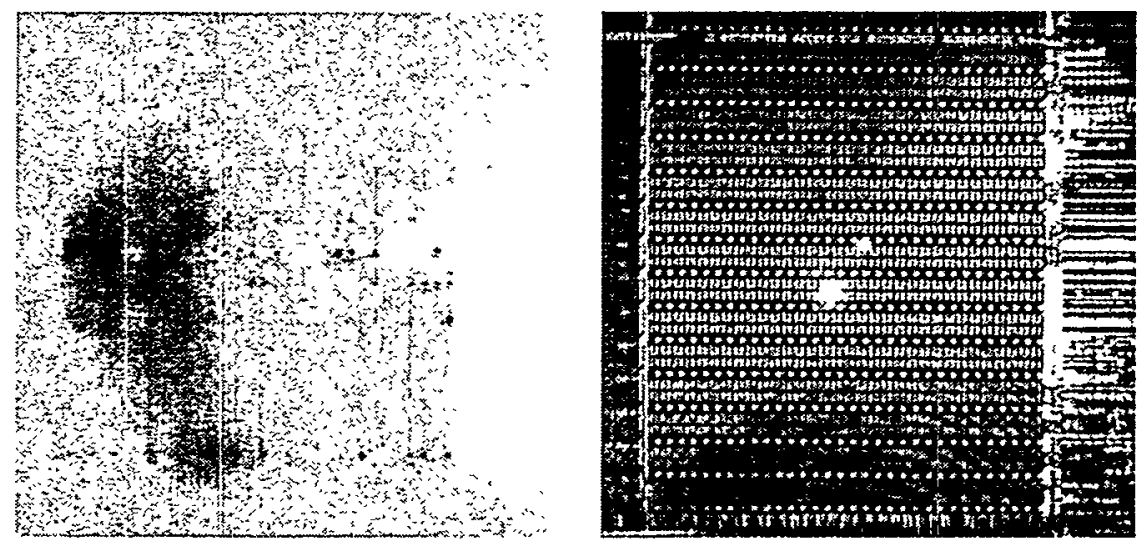

Fig. 32. Higher magnification backside TIVA (left) and reflected (right) images of the suspected capacitor damage shown in Fig. 31.

Fig. 33 is an example showing a small interlevel short on a $0.5 \mu \mathrm{m}$ SRAM from the front side. The dark line in the TIVA image should be electrically continuous but abruptly stops at the site indicated by the arrow. A FIB cross section of this area revealed an interlevel short at the site of the abrupt contrast change as shown in Fig. 34.

A final front side TIVA example of an ESD damage site is shown in Fig. 35. The ESD damage resulted in an input to VDD short and could be seen in a TIVA image of the whole die. The IC was susceptible to ESD because of mask misalignment, resulting in metal over gate oxide. The short site is the dark dot in the TIVA image. This TIVA image is significant because no bias was applied during image acquisition. The voltage variations were generated by a thermocouple effect across the metal-silicon alloy. With no bias applied the TIVA technique is very sensitive to thermocouple-type shorts. 

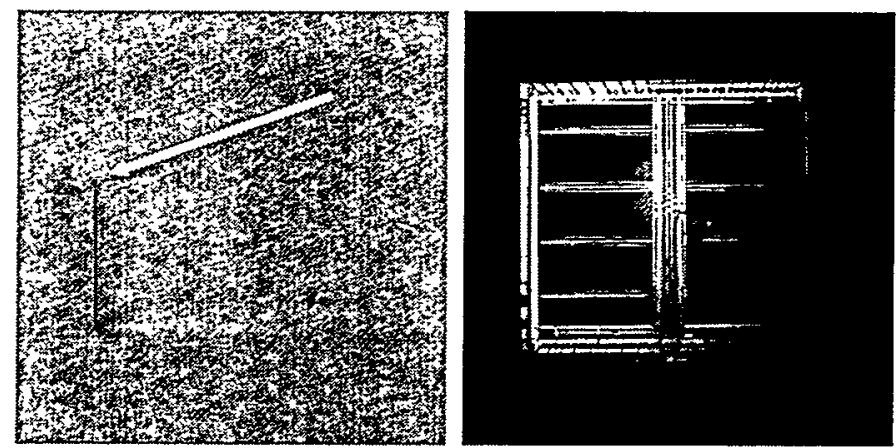

Fig. 33. Front side TIVA (left) and reflected (right) images of a shorted conductor. The arrow identifies the short site.

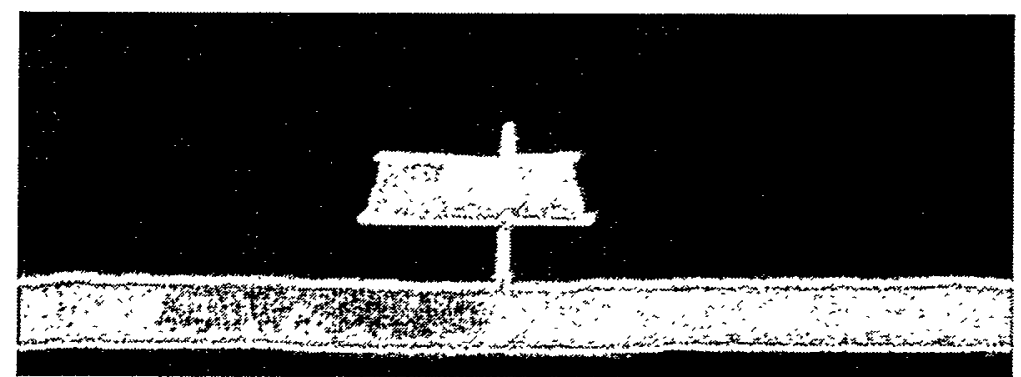

Fig. 34. FIB cross section of the short site identified in Fig. 33 revealing a shorting filament.
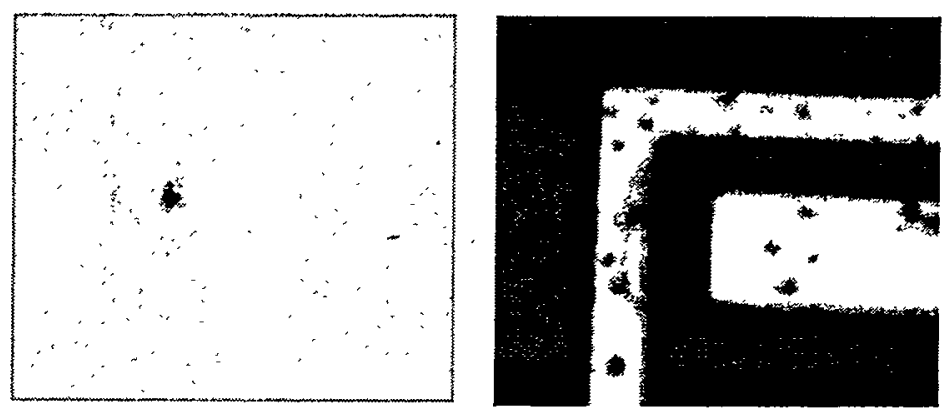

Fig. 35. TIVA (left) and reflected (right) images of ESD damage (dark spot).

The significant temperature variations at $1 \mathrm{MHz}$ predicted by the modeling results in Section 7 above and Section 9 below indicate that a lock-in approach with a pulsed beam might be applicable to TIVA/SEI analysis. In practice, the lock-in approach with the pulsed beam was not successful with our present system. The Keithley 236 constant current supply that was used for TIVA/SEI measurements has too limited a bandwidth to respond to a $1 \mathrm{MHz}$ pulsed beam. Decreasing the pulse frequency is not practical. If the pulses are reduced to a frequency much below $1 \mathrm{MHz}$ to accommodate the Keithley 236 bandwidth, the scan speed of the laser in our 
LSM must also be decreased beyond the system's slowest speed so that the dwell time of the laser at each pixel in the image is much larger than the pulse width of the laser

\section{Extended Thermal Modeling and TIVA Analysis of a Polysilicon-Metal Test Structure}

\subsection{Experimental Procedures for Analysis of the Test Structure}

All TIVA measurements were preformed on the improved system described above. Typical scan times were 4 to 8 seconds per frame. Line averaging of 4 to 8 lines was also used to improve the signal-to-noise ratio in TIVA images.

An optical image of the test structure is shown in Fig. 38. The test structure consists of two heavily doped polysilicon lines that are $4 \mu \mathrm{m}$ wide and $4 \mu \mathrm{m}$ apart. Each polysilicon line is electrically connected in a serpentine pattern. Over $90 \%$ of one polysilicon line is insulated from the silicon substrate by a $0.5-\mu \mathrm{m}$ thick $\mathrm{SiO}_{2}$ layer with the rest of the line insulated by a $200-\AA$ thick $\mathrm{SiO}_{2}$ layer. Over $90 \%$ of the other polysilicon line is insulated by a $200-\AA$ thick $\mathrm{SiO}_{2}$ layer with the rest of the line insulated by a $0.5-\mu \mathrm{m}$ thick $\mathrm{SiO}_{2}$ layer. A $0.5-\mu \mathrm{m}$ thick $\mathrm{SiO}_{2}$ layer overlays these two polysilicon lines. On top of this oxide layer are two aluminum lines that are 4 $\mu \mathrm{m}$ wide and $4 \mu \mathrm{m}$ apart. The aluminum lines are orthogonal to the polysilicon lines. Each aluminum line is also connected in a serpentine fashion. A 1- $\mu$ m thick $\mathrm{SiO}_{2}$ passivation layer overlays these aluminum lines. Focused ion beam (FIB) cross sections of this polysilicon-metal test structure are shown in Figs. 39 and 40.

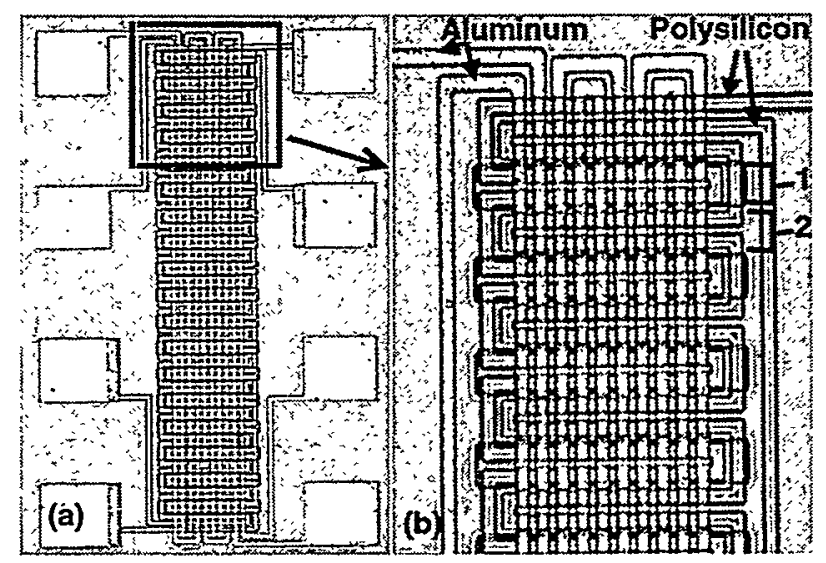

Fig. 38. Low-magnification (a) and high-magnification (b) front side optical images of the polysilicon-metal test structure used in TIVA measurements. In areas 1 and 2, the polysilicon is insulated from the silicon substrate by $0.5-\mu \mathrm{m}$ thick and $200-\AA$ thick $\mathrm{SiO}_{2}$ layers respectively.

The test structure was mounted on a dual inline package (DIP). Front side measurements focused the scanned laser beam through the 1- $\mu \mathrm{m}$ thick $\mathrm{SiO}_{2}$ passivation layer onto either the aluminum or polysilicon lines. The backside of the DIP package was mechanically ground and polished to allow optical access from the back of the die. The laser beam was focused through the $500-\mu \mathrm{m}$ thick silicon substrate onto either the polysilicon or aluminum lines. 
Either the aluminum or polysilicon lines were biased with a Keithley Instruments 236 or 238 in a constant current configuration. The resistance of the aluminum line was $\sim 30 \Omega$ and the typical current and voltage during TIVA measurements were $50 \mathrm{~mA}$ and $1.5 \mathrm{~V}$. The resistance of the polysilicon line was $\sim 17000 \Omega$ and typical current and voltage were $0.8 \mathrm{~mA}$ and $13.6 \mathrm{~V}$. As the focused laser beam scanned across the test structure, temperature increased due to localized laser heating. The heating increased the resistance of the aluminum or polysilicon lines and the TIVA image was formed by measuring the ac coupled variation in the power supply voltage $\left(V_{D D}\right)$. The polarity of the voltage amplifier was set up such that the darkest area in the TIVA images indicated the largest resistance rise, indicating the largest change in temperature.

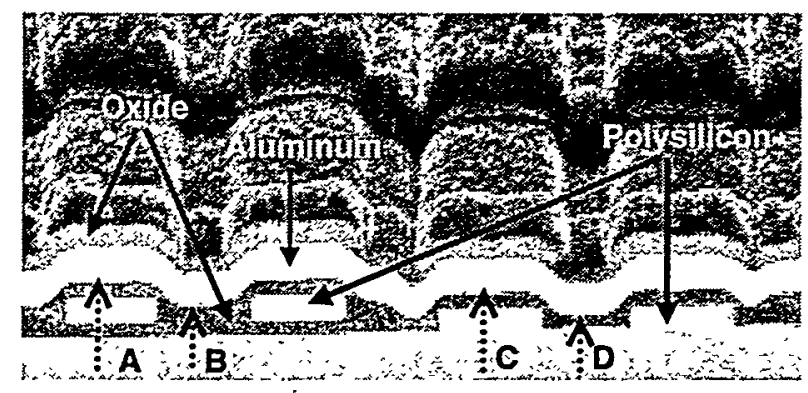

Fig. 39. Focused ion beam cross-section image of the polysilicon-metal test structure along the aluminum line. The dashed arrows indicate the areas of backside laser illumination on the aluminum line. Local temperature rises due to laser heating in areas A, B, C, and D are different, resulting in variation in TIVA signals along the biased aluminum line.

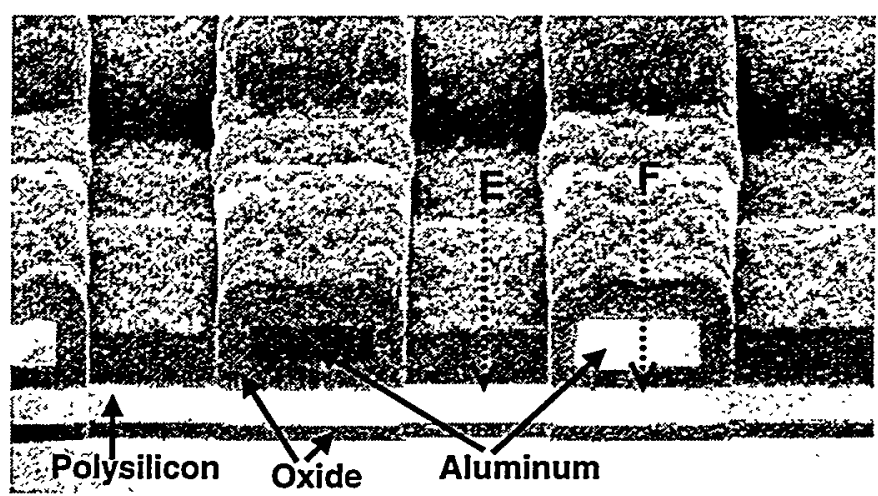

Fig. 40. Focused ion beam cross-section image of the polysilicon-metal test structure along the polysilicon line. The dashed arrows indicate the areas of front side laser illumination on the polysilicon line. Local temperature rises due to laser heating in areas $\mathrm{E}$ and $\mathrm{F}$ are very different, resulting in variation in TIVA signals along the biased polysilicon line.

\subsection{Thermal Modeling and Simulations of the Test Structure}

Thermal profiles of the test structure resulting from the small-area laser heating were simulated with TAS, a commercial finite difference solver [16]. The 3-dimensional steady state heat 
transfer equation was solved subject to the appropriate boundary conditions. The solver worked in an interactive visual environment to generate a mesh geometry. This mesh geometry, coupled with specified material conductivity properties, heat inputs, and boundary conditions gave a detailed description of temperatures within the test structure. A model was constructed with brick elements describing the material shapes of the test structure. The brick elements with orthogonal edges had their heat transfer described by twelve internode thermal resistance, $R_{i j}$. $R_{i j}$ can be written as

$$
R_{i j}=\frac{\Delta x_{i j}}{k \times A_{i j}},
$$

where $\Delta x_{i j}$ is the spacing between nodes $i$ and $j ; k$ is the thermal conductivity and $A_{\mathrm{ij}}$ is the cross sectional area of the brick element. When the brick elements had angles other than 90 degrees or when anisotropic material properties were used, additional diagonal resistances between the nodes were added. Heat inputs and fixed temperature boundary conditions were then assigned to specific nodes. Temperature was solved iteratively at each unconstrained node with the finite difference solving routine. A steady-state solution was determined using,

$$
T_{u i}=T_{i} \times(1-\beta)+\beta \times \frac{\sum_{j}\left(\frac{T_{j}}{R_{i j}}\right)+Q_{i}}{\sum_{j} \frac{1}{R_{i j}}},
$$

where $T_{u i}$ is the updated temperature for $i^{\text {th }}$ node and is related to the previous temperature, $T_{i} . \beta$ (typically $1.5-1.9$ ) is a parameter selected to optimize convergence of the solution. $Q_{i}$ is the heat input to the node. $T_{u i}$ is iterated until the solution converges to generate a maximum $\Delta \mathrm{T}$ below some convergence criteria, typically $10^{-4}{ }^{\circ} \mathrm{C}$. This finite difference method was efficient and converged to give solutions for 25,000 brick elements in about 20 seconds on a $400-\mathrm{MHz}$ Window-based PC computer.

A model composed of brick elements $1 \times 1 \mu \mathrm{m}$ in surface area and varying thickness was used to simulate the temperatures in areas A, B, C, and D of the test structure shown in Fig. 39. In this model, aluminum and polysilicon lines were set to be $4 \mu \mathrm{m}$ wide, $1 \mu \mathrm{m}$ thick, and in a non planarized geometry. In area $\mathrm{A}, 0.5-\mu \mathrm{m}$ thick oxide layers were used above and below the polysilicon lines. In area $C, 0.5-\mu \mathrm{m}$ and $200-\AA$ thick oxide layers were used above and below the polysilicon line respectively. In areas $B$ and $D, 1-\mu \mathrm{m}$ and $0.5-\mu \mathrm{m}$ thick oxide layers were used under the aluminum in the region between polysilicon lines respectively. A $24 \times 64 \mu \mathrm{m}$ segment of the test structure on a $17 \mu \mathrm{m}$ thick layer of silicon substrate was also used in the model. The back face of the substrate was maintained at the base temperature of $25^{\circ} \mathrm{C}$. Heat was input to a $1 \times 1 \mu \mathrm{m}$ area on the lower surface of the aluminum line to simulate backside laser heating. Additional heat was added to the polysilicon lines to simulate laser absorption in these lines. A plane normal to the die surface and aligned with the center of one of the aluminum lines was used to display the simulated temperature profiles.

A thermal conductivity value of $1.48 \mathrm{~W} / \mathrm{cm}^{-}{ }^{\circ} \mathrm{C}$ was used for the silicon substrate and polysilicon lines [18]. A value of $1.06 \mathrm{~W} / \mathrm{cm}^{\circ}{ }^{\circ} \mathrm{C}$ was used for aluminum. The aluminum value was about $30 \%$ below that for bulk aluminum listed in reference 15 , but was selected to match the measured electrical conductivity of aluminum lines in the test structure. A thermal conductivity value 
of $0.011 \mathrm{~W} / \mathrm{cm}-{ }^{\circ} \mathrm{C}$ was used for thin oxide films [19]. All thermal conductivity values were assumed constant with temperature. Convection and radiation were not included in the simulations and thermal profiles were calculated based solely on thermal conduction.

\subsection{Test Structure Results}

Fig. 41 shows the backside TIVA image of a biased aluminum line in the test structure. Areas A, B, C, and D in Fig. 41 correspond to the same areas shown in Fig. 39. Area A has a polysilicon conductor placed directly beneath the aluminum line and shows the strongest TIVA contrast (darkest area). The polysilicon line in this area is insulated from the silicon substrate by a $0.5-$ $\mu \mathrm{m}$ thick $\mathrm{SiO}_{2}$ layer. The physical structure in area $\mathrm{B}$ is similar to area $\mathrm{A}$ except there are no polysilicon conductor beneath the aluminum lines. The polysilicon conductor in area $\mathrm{C}$ is directly beneath the aluminum, but the polysilicon is insulated from the silicon substrate by a $200-\AA$ silicon dioxide layer. Area $D$ is similar to area $C$ except there is no polysilicon conductor underneath the aluminum line. The bright bands at the right of the dark TIVA signals in Fig. 41 are amplifier artifacts due to the overshoot of amplifier response and are not related to any temperature change in the structures.

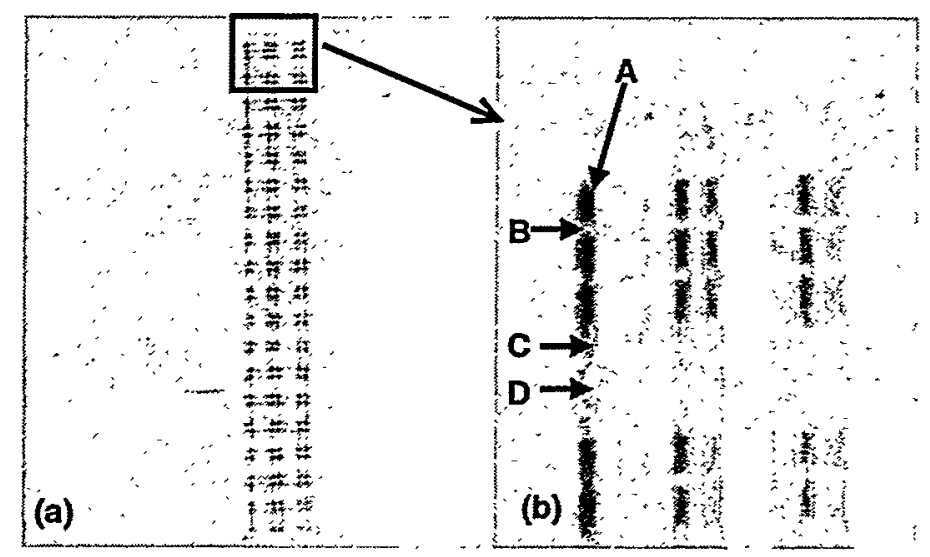

Fig. 41. Backside TIVA low-magnification (a) and high-magnification (b) images of a biased aluminum line in the polysilicon-metal test structure. Areas A, B, C, and D correspond to the same areas shown in Fig. 39.

Thermal simulations were performed to help explain the backside TIVA image of the aluminum line in Fig. 41. Initially, absorption in both the polysilicon conductors and silicon substrate was assumed to be negligible. Since aluminum is highly reflective at infrared wavelengths [20], $97 \%$ of the laser beam was assumed to reflect from the aluminum and only $3 \%$ of the laser power ( $0.6 \mathrm{~mW}$ out of the total laser power of $20 \mathrm{~mW}$ ) was absorbed by the aluminum line in a $1 \mu \mathrm{m}^{2}$ area. The simulation results in Fig. 42 show the temperature profiles on a plane cutting normal to the die surface and aligning with the center of aluminum line. The peak temperatures for areas $\mathrm{A}$ and $\mathrm{B}$ were $31.19^{\circ} \mathrm{C}$ and $31.24^{\circ} \mathrm{C}$ respectively assuming the base temperature on the die to be $25^{\circ} \mathrm{C}$. This small difference in peak temperatures with position of the heat input was a result of heat spreading along the aluminum lines over an area that was much larger than the scale of the test pattern. The heat spreading distance was at least 3 times larger than the width of the 
aluminum line (Fig. 42). The location of the laser probe beam relative to line geometry, therefore, did not affect the peak temperature.
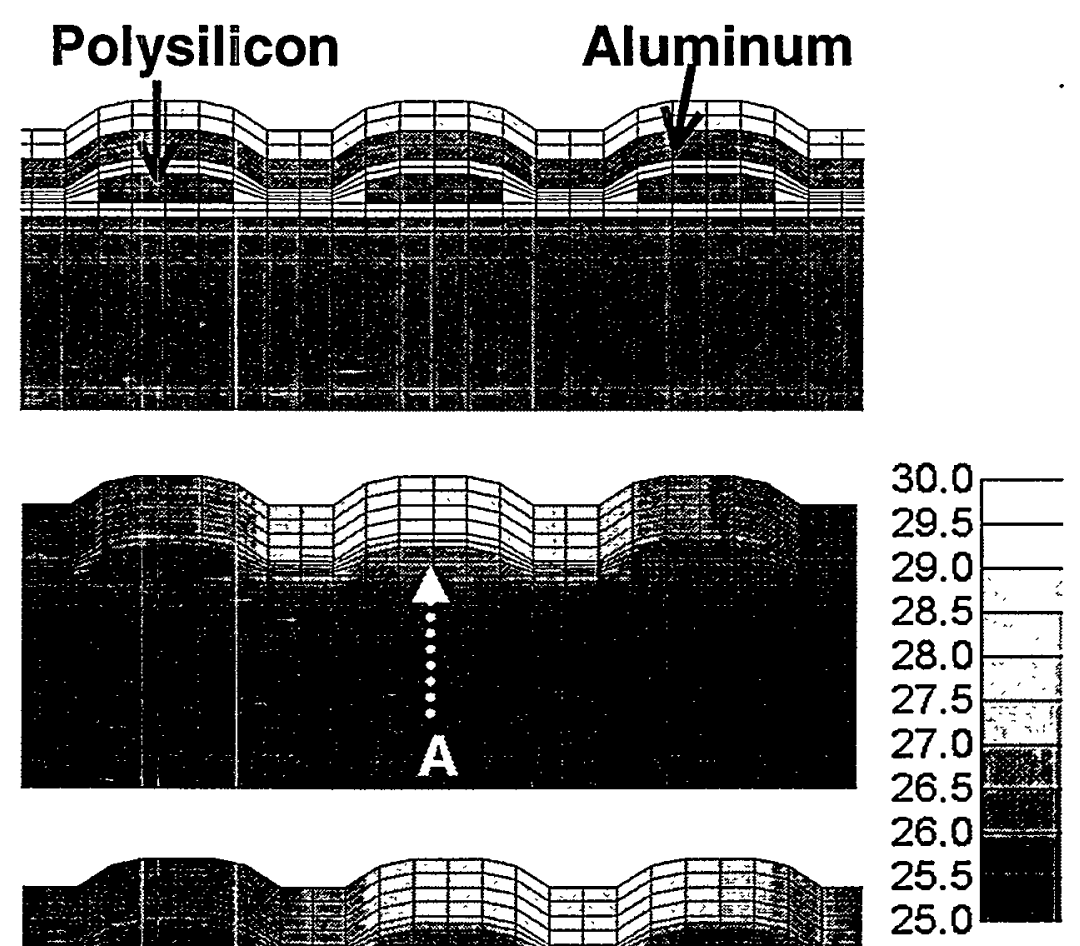

Fig. 42. The lattice structure (top) used in the simulation of temperature profiles at areas A and $\mathrm{B}$ in the test structure. Calculated temperature profiles for the same absorbed power $(0.6 \mathrm{~mW})$ on an aluminum line at area $\mathrm{A}$ (middle) and $\mathrm{B}$ (bottom) in the lattice structure. The peak temperatures were $31.19^{\circ} \mathrm{C}$ and $31.24^{\circ} \mathrm{C}$ for areas $\mathrm{A}$ and $\mathrm{B}$ respectively, relative to a base die temperature of $25^{\circ} \mathrm{C}$.

This lack of temperature variation with beam location in Fig. 42, assuming constant power absorption at areas A and B, was inconsistent with TIVA data in Fig. 41. Therefore, the model was improved to include laser absorption by the polysilicon beneath the metal. The absorption coefficient of polysilicon at $1.3 \mu \mathrm{m}$ was determined from Fig. 43, a plot of absorption coefficient versus resistivity for several reference silicon wafers. The absorption coefficient of polysilicon was determined to be $530 \mathrm{~cm}^{-1}$ corresponding to the measured resistivity of $1.59 \times 10^{-3} \mathrm{ohm}-\mathrm{cm}$. This absorption coefficient indicated about $5 \%$ of laser beam was absorbed in the polysilicon as the beam was focused onto the aluminum lines in area A. Aluminum lines are highly reflective 
at this wavelength and most of the incoming laser beam (97\%) was reflected off the aluminum surface with only about $3 \%$ of beam energy absorbed in the aluminum line. About $5 \%$ of the reflected laser beam was again absorbed by the polysilicon. The total polysilicon absorption from the incoming and reflected laser beams was about $10 \%$ of the incoming laser intensity. In area $\mathrm{B}$, there is no polysilicon beneath the aluminum line and all absorption takes place in the aluminum.

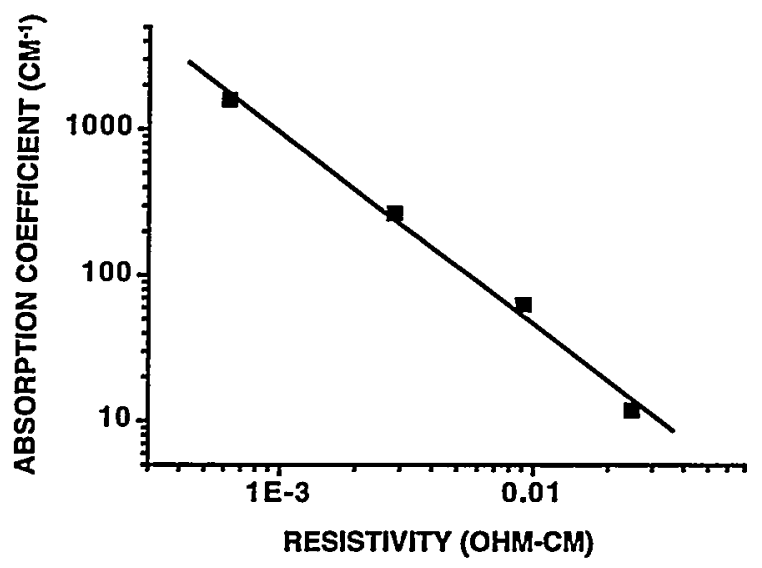

Fig. 43. Absorption coefficient versus resistivity for several silicon wafers. This curve was used to estimate the absorption coefficient of polysilicon lines in the test structure.

Fig. 44 shows the results of temperature calculations at areas A and B using the improved thermal model that includes the absorbed power difference. The peak temperature at area $\mathrm{A}$ was calculated to be $37.3^{\circ} \mathrm{C}$ relative to a base temperature on the die of $25^{\circ} \mathrm{C}$ in contrast to $30.2^{\circ} \mathrm{C}$ at area B. Thermal modeling was also performed on areas $C$ and $D$ with the underlying $200-\AA$ thick oxide. The peak temperatures were calculated to be 30.5 and $29.5^{\circ} \mathrm{C}$ for areas $\mathrm{C}$ and D respectively. Thermal simulation results show that area $\mathrm{A}$ has the largest rise in local temperature due to the largest absorption of laser power. This rise in temperature leads to a significant increase in the aluminum resistance, giving the largest contrast in the TIVA image (Fig. 41).

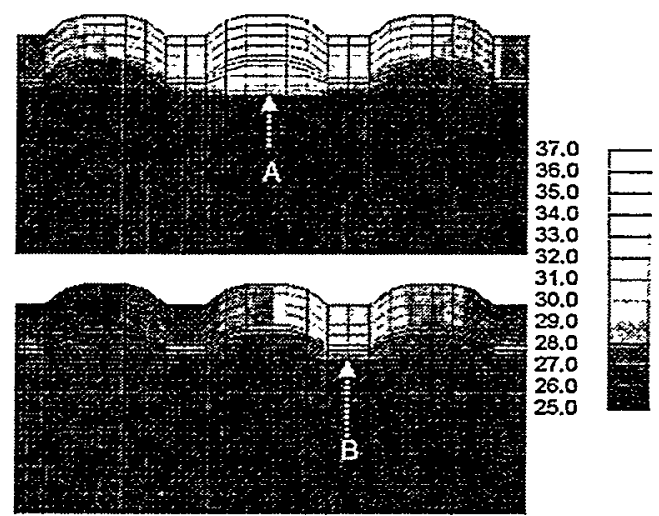

Fig. 44. Calculated temperature profiles of an aluminum line at area A (top) and area B (bottom) based on an improved model that included laser heating of the polysilicon beneath the aluminum. The peak temperatures were $37.3^{\circ} \mathrm{C}$ and $30.2^{\circ} \mathrm{C}$ for areas $\mathrm{A}$ and $\mathrm{B}$ respectively, relative to a base die temperature of $25^{\circ} \mathrm{C}$. 
The difference in peak temperature between areas $\mathrm{A}$ and $\mathrm{C}$ is due to the variation in $\mathrm{SiO}_{2}$ thickness beneath the polysilicon line. To understand this difference, the temperature rise was calculated as a function of the oxide thickness on a series of aluminum lines insulated from the silicon substrate by a $\mathrm{SiO}_{2}$ layer. The aluminum lines were $4 \mu \mathrm{m}$ wide, $1 \mu \mathrm{m}$ thick, and spaced on an $8 \mu \mathrm{m}$ pitch. The aluminum line was heated in a $1 \mu \mathrm{m}^{2}$ area on the lower side with a laser input of $0.6 \mathrm{~mW}$. The $\mathrm{SiO}_{2}$ layer thickness was varied in a series of calculations and the peak temperature was recorded for each case (Fig. 45). For thin oxides ( $<0.2 \mu \mathrm{m}$ in thickness), there was little heat spread along the aluminum line as heat spread occurred in a direction toward substrate; consequently, the peak temperature increased sharply with increasing oxide thickness. For thick oxides $(>0.2 \mu \mathrm{m})$, there was significant heat spread along the aluminum line, resulting in a much smaller temperature rise per unit change in oxide thickness as compared with the thin layer.

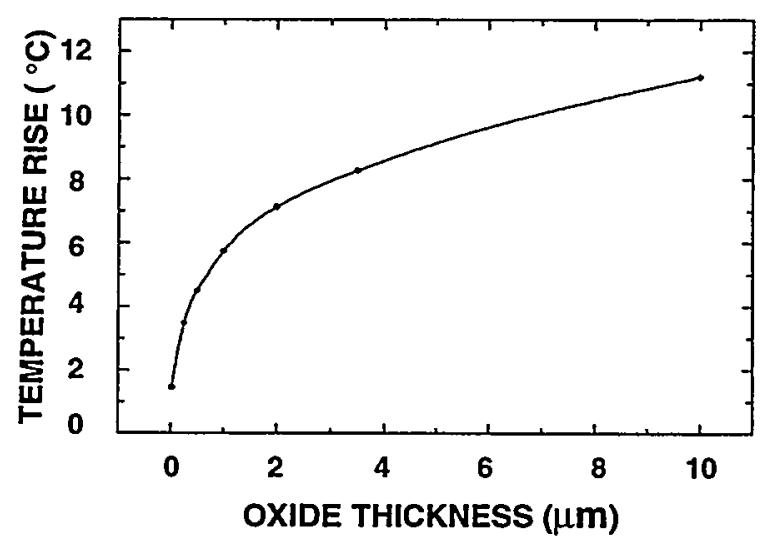

Fig. 45. Peak temperature rise on an aluminum line insulated from the silicon substrate by a $\mathrm{SiO}_{2}$ layer plotted as a function of the $\mathrm{SiO}_{2}$ thickness.

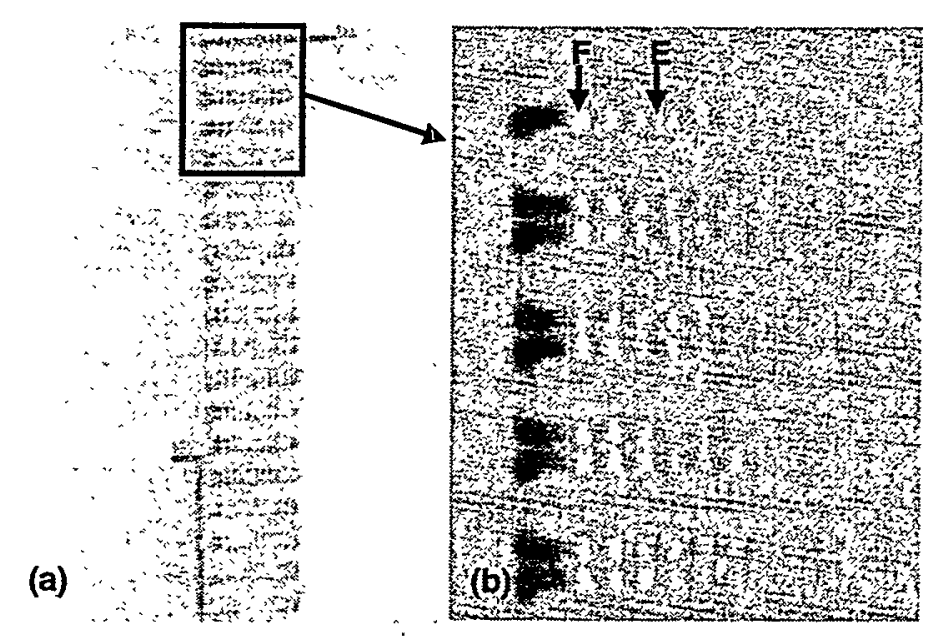

Fig. 46. Front side TIVA low-magnification (a) and high-magnification (b) images of a biased polysilicon line with over $90 \%$ of the line insulated from silicon substrate by a $0.5-\mu \mathrm{m}$ thick $\mathrm{SiO}_{2}$ layer. Areas $\mathrm{E}$ and $\mathrm{F}$ correspond to the same areas shown in Fig. 40. 
The differences in peak temperature as a function of $\mathrm{SiO}_{2}$ thickness are illustrated in Figs. 46 and 47 that show the front side TIVA images of the biased polysilicon lines. The TIVA signals are much stronger in Fig. 46 where the polysilicon line is insulated from the silicon substrate by a $0.5-\mu \mathrm{m}$ thick $\mathrm{SiO}_{2}$ layer and much weaker in Fig. 47 where the polysilicon line is insulated from the silicon substrate by a $200-\AA$ thick $\mathrm{SiO}_{2}$ layer. Fig. 46 also shows variation in TIVA contrast along the polysilicon line. This variation is due to preferential laser absorption, similar to that observed for the backside aluminum described in previous paragraphs. In area $\mathrm{E}$ (Fig. 46), the laser was focused onto the polysilicon line and about $5 \%$ of laser beam was directly absorbed by the polysilicon. In area $\mathrm{F}$, the aluminum line absorbed about $3 \%$ of the laser beam and the polysilicon in this area was heated only indirectly through thermal conduction from the aluminum line. These differences in laser absorption cause a more significant temperature rise in area $\mathrm{E}$ than in area $\mathrm{F}$, leading to stronger TIVA signals in area $\mathrm{E}$.

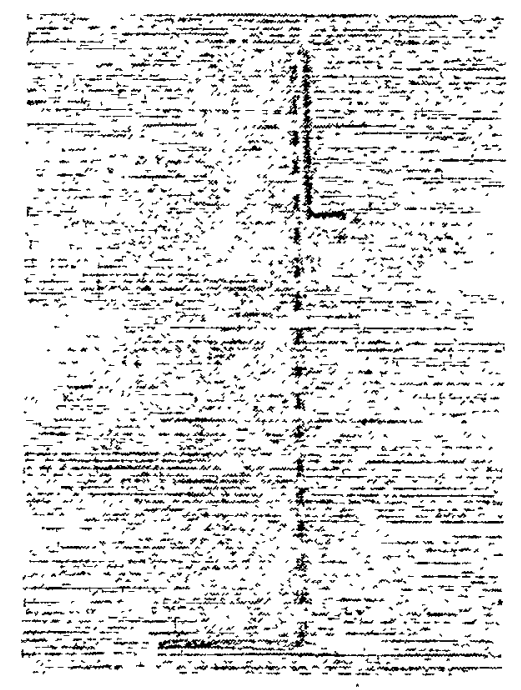

Fig. 36. Front side TIVA image of a biased polysilicon line with over $90 \%$ of the line insulated from silicon substrate by a $200-\AA$ thick $\mathrm{SiO}_{2}$ layer. Weak TIVA signals were observed in this line except in the area where the polysilicon was insulated from the silicon substrate by a $0.5-\mu \mathrm{m}$ thick $\mathrm{SiO}_{2}$ layer.

\subsection{Pulsed Laser Modeling}

Our final modeling work examined the heating effects of surface structures with a pulsed laser. For modeling purposes an aluminum line was assumed to be separated from the Si substrate by a $1 \mu \mathrm{m}$ thick oxide layer. Additionally, a $0.5 \mu \mathrm{m}$ thick layer of oxide on top of the aluminum line was included. The aluminum line was also assumed to be $0.5 \mu \mathrm{m}$ thick and $1 \mu \mathrm{m}$ wide. The thermal response at the bottom surface of the aluminum line and at the Si/oxide interface were calculated assuming a $1 \mathrm{MHz}, 50 \%$ duty cycle, $10-\mathrm{mW}$ pulsed laser was applied at these two locations (Fig. 48). Since the aluminum line is thermally insulated from silicon by a layer of oxide, a fixed amount of laser power produces a much larger temperature change compared to the heating at Si/oxide interface. In addition, the time required for heat to diffuse through the oxide layer slows the frequency response of temperature variations on these layered features. Note that the thermal response is much slower in Fig. 48 compared to Fig. 25, but significant temperature variations are predicted. 


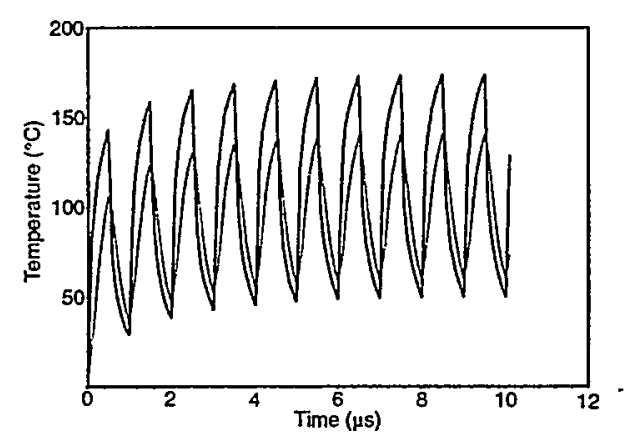

Fig. 48. Thermal responses to a $1 \mathrm{MHz}$ pulsed laser heating at the bottom surface of the $\mathrm{Al}$ line (larger amplitude) and at Si/oxide interface (smaller signal).

\section{Conclusions}

The SEI and TIVA techniques have been developed as new, nondestructive analytical methods for backside failure analysis. Through backside localization of open interconnections, SEI provides what has been a critical "missing link" in IC analysis capability. The high sensitivity of TIVA yields a practical tool for short localization from both the front and backside of the IC.

Thermal modeling and simulations account for the unique thermal profiles of a polysilicon-metal test structure generated by TIVA. Variation in laser absorption is the main factor affecting changes in local heating and temperature rise in different locations on the test structure. Differences of oxide thickness in the test structure also affect the variation of local heat conduction and temperature. Thermal modeling shows that the local variation in heat conduction is less important than the absorbed laser power in determining the local temperatures because the present test structure has feature sizes that are small compared to the length over which heat spreads. Thermal simulations provide a better understanding of the TIVA thermal profiles and this understanding facilitates the use of TIVA technique in localizing defects within the complex IC structures.

The improvements to the SEI and TIVA image acquisition system have produced a practical, robust, non-destructive method for rapidly localizing open and shorted interconnections on ICs from the front and backside of the device. The examples given demonstrate the effectiveness of the technique for defect localization. The increased system sensitivity provides powerful new analytical tools for failure analysis.

\section{References}

[1] D.L. Barton, P. Tangyunyong, J.M. Soden, A.Y. Liang, F.J. Low, A.N. Zaplatin, K. Shivanandan, and G. Donohoe, "Infrared Light Emission From Semiconductor Devices", ISTFA, 1996, pp. 9-17.

[2] J.C Tsang and J.A. Kash, Appl. Phys. Lett., 70., 1997 pp. 889-891.

[3] E.I. Cole Jr., J.M. Soden, J.L. Rife, D.L. Barton, and C.L. Henderson, "Novel Failure Analysis Techniques Using Photon Probing in a Scanning Optical Microscope", IRPS, 1994, pp. 388-398. 
[4] M. Paniccia, T. Eiles, V.R.M. Rao, and W.M. Yee, "Novel optical probing technique for flip chip packaged microprocessors," ITC, 1998, pp. 740-747.

[5] K. Nikawa and S. Inoue, "Various Contrasts Identifiable From the Backside of a Chip by $1.3 \mu \mathrm{m}$ Laser. Beam Scanning and Current Changing Imaging”, ISTFA, 1996, pp. 387392.

[6] N.W. Ashcroft and N.D. Mermin, Solid State Physics, Philadelphia, Saunders College, 1976, ch. 1, pp. 24-25.

[7] Internet site, http://www.seebeck.com/seebeff.html, referenced 1/7/98.

[8] T. Koyama, Y. Mashiko, M. Sekine, H. Koyama, and K. Horie, "New Non-Bias Optical Beam Induced Current (NB-OBIC) Technique for Evaluation of Al Interconnects", IRPS, 1995, pp. 228-233.

[9] E.I. Cole Jr. and R.E. Anderson, "Rapid Localization of IC Open Conductors Using Charge-Induced Voltage Alteration (CIVA)," IRPS, 1992, pp. 288-298.

[10] E.I. Cole Jr., J.M. Soden, B.A. Dodd, and C.L. Henderson, "Low Electron Beam Energy CIVA Analysis of Passivated ICs," ISTFA, 1994, pp. 23-32.

[11] E.I. Cole Jr., J.M. Soden, P. Tangyunyoug, P.L. Candelaria, R.W. Beegle, D.L. Barton, C.L. Henderson, and C.F. Hawkins, "Transient Power Supply Voltage $\left(v_{D D T}\right)$ Analysis for Detecting IC Defects," ITC, 1997, pp. 23-31.

[12] R.A. Serway, Physics for Scientists and Engineers with Modern Physics, second edition, Saunders Publishing Co., NY, 1986, ch. 27, pp. 605-606.

[13] S. Ferrier, "Thermal and Optical Enhancements to Liquid Crystal Hot Spot Detection Methods," ISTFA, 1997, pp. 57-62.

[14] S.E. Aw, H.S. Tan, and C.K. Ong, "Optical Absorption Measurements of Band-gap Shrinkage in Moderately and Heavily Doped Silicon", J. Phys.: Condens. Matter, 3, 1991, pp. 8213-8223.

[15] D.L. Barton and P. Tangyunyong, "Fluorescent Microthermal Imaging - Theory and Methodology for Achieving High Thermal Resolution Images," Microeleronic Engineering, 31, 1996, pp. 271-279.

[16] Thermal Analysis System, TAS, Harvard Thermal Inc., Harvard MA 01451.

[17] C.L. Henderson, J.M. Soden, and C.F. Hawkins, "The Behavior \& Testing Implications of CMOS IC Open Circuits," ITC, 1991, pp. 302-310.

[18] J. Sergent and A. Krum, Thermal Management Handbook for Electronic Assemblies, (McGraw Hill, New York, 1998).

[19] M. B. Kleiner, S. A. Kuhn, and W. Weber, IEEE Trans. on Electron Devices 43, 1602 (1996).

[20] J. M. Palmer, Handbook of Optics, Volume II, $\cdot 2^{\text {nd }}$ Edition, edited by M. Bass, E. W. Van Stryland, D. R. Williams and W. L. Wolfe (McGraw Hill, New York, 1995), Chap. 25. 


\section{EVALUATION OF PROGRESS TOWARDS GOALS}

The goal of the LDRD was to develop and optimize techniques to localize open and shorted interconnections from the backside of an IC for the purposes of failure analysis, yield improvement, and new process technology development. In this two-year project, the first year was intended to demonstrate the technical soundness of the TIVA and SEI approaches on simple devices and show that the techniques could be applied to state-of-the-art ICs. The second year was to focus on refinements and optimization of sample preparation and technique implementation. Our original milestones were:

\section{FY98}

1. Demonstrate technical soundness of SEI and SL (short localization) on complex Sandia ICs from the front side.

2. Develop and verify simple models of thermal deposition and diffusion, conductor potential changes in response to changes in temperature, background temperature, and conductor material.

3. Develop backside sample preparation techniques and demonstrate the technical soundness on Sandia ICs from the backside.

4. Develop signal optimization techniques by changing the die temperature and optimizing the SOM efficiency at the SEISL wavelength.

5. Demonstrate technical soundness on commercial state-of-the-art ICs from the front and backside.

6. Publish preliminary results.

\section{FY 99}

7. Develop backside preparation thinning while maintaining electrical operation.

8. Develop models of transistor and conductor response to pulsed laser heating and demonstrate signal optimization using a pulsed beam and lock-in techniques.

9. Demonstrate the limitations of through metal probing and establish best practices guidelines for using the optimization techniques developed.

10. Publish final results.

11. Produce reviewed and approved SAND report in September 1999.

Overall the technique development has been very successful, with TIVA and SEI becoming standard tools in Sandia's failure analysis laboratory.

By the end of the FY98 the progress toward the milestones was as follows:

Milestones 1. \& 5. These milestones have been completed as SEI and SL images have been demonstrated from the front and backside of Sandia and commercial ICs. The SEI images produced are the only known method to directly detect and localize open interconnections form the backside of an IC and the SL images show much greater sensitivity than previous methods.

Milestone 2. The results of thermal modeling showed that the focused $1340 \mathrm{~nm}$ laser used for SEI and SL produced about a $1{ }^{\circ} \mathrm{C} / \mathrm{mW}$ temperature change in a silicon substrate. The 
system used for the LDRD studies has a $120 \mathrm{~mW}$, single mode, polarized laser used for thermal stimulus. The transmission efficiency of our scanning optical microscope (SOM), about $3 \%$ to $25 \%$ depending upon the objective lens used, indicates that thermal gradients on the order of $4{ }^{\circ} \mathrm{C}$ to $30^{\circ} \mathrm{C}$ can be produced, creating potential variations in the 1 's to 10 's of $\mu \mathrm{V}$ on open conductors via the Seebeck Effect. These are the potential variations observed in SEI images.

Modeling indicated that because of the high thermal conductivity of silicon, thermal gradients would be produced locally only at the point where the laser is focused and not throughout the IC die, reducing concerns about substrate heating from the scanned laser stimulus. Modeling work has also indicated that silicon's high thermal conductivity will permit pulsed laser SEI and SL analysis. The simulation results show that a pulsed laser stimulus at a frequency of $1 \mathrm{MHz}$ (50\% duty cycle) will generate temperature differences of approximately $90 \%$ of the continuous exposure beam between pulses. This result indicates that the pulsed laser experiments planned for next year will generate ac changes in temperature that can be used for improving the SEI and SL signal strength.

Milestone 3. This milestone was completed and is demonstrated by the backside SEI and SL images produced. Grinding and polishing of packaged ICs required using an epoxy to support the front side of the IC die. To maintain IC functionality the stresses of the epoxy on the IC must be minimal during the curing process. Several different epoxies were examined with the best results obtained using an epoxy that does not heat while curing.

Presently precision milling equipment is being explored as a possible improvement to the grinding/polishing approach used thus far. The initial results are promising with decreased preparation time ( 1 hour compared to the $1 / 2$ day with the present system) and a higher quality polish.

Milestone 4. The SEI and SL signal strengths are relatively weak compared to other failure analysis methods. The images produced so far, while demonstrating feasibility, have required significant averaging. Two approaches outlined for improving the signal strength explored in this first year were sample heating and optimization of the SOM transmission efficiency. Global heating of the die produced no improvement in the SEI or SL signals. This is consistent with the thermal modeling results of Milestone 2. The transmission efficiency of the SOM was improved greatly (about $80 \mathrm{X}$ ) by modifying the laser beam path in our microscope to by-pass inefficient (but flexible) beam combination elements. The manufacturer of our SOM, Zeiss, provides a fiber optic connection that by-passes the inefficient elements. We used the connection port with a free space laser (no fiber used) to achieve increased laser intensity to the sample. The improvements generated stronger signals for SEI and SL.

An additional system improvement not planned for in the original LDRD was an approved capital pool item for FY 98, a higher power $350 \mathrm{~mW}$ laser. The existing system used a 120 $\mathrm{mW}$ laser. Higher power lasers are available but they are relatively expensive $(>\$ 40 \mathrm{~K})$ and there are limits to the power that can be transmitted through the SOM optics without 
damaging the surface coatings. The new laser has been installed and is showing enhanced SEI and SL image acquisition.

Milestone 6. The preliminary results of the LDRD were presented at the International Reliability Physics Symposium (IRPS) in April 1998. The paper was very well received with one IC manufacturer interested in pursuing a licensing agreement to use SL and SEI and an equipment manufacturer expressing interest in licensing SEI and SL on their line of SOMs. As a result of the IRPS publication Sandia has been asked to provide an invited paper on SEI and SL in the journal Microelectronic Engineering.

The end of FY 99 was highlighted by the following progress:

Milestone 7. Thinned samples were provided by external customers interested in knowing if the SEI/TIVA analysis techniques would be useful for their advanced technologies from the backside of IC dice. By thinning the samples the amount of heat delivered to defects is increased, and hence the SEI and TIVA signals highlighting the defects are increased. This proved to be true for the samples examined. Additionally, the application of an antireflective, infrared coating to the backside of the sample increased the heat delivery by about $2 \mathrm{X}$.

Milestone 8. Modeling analysis showed how the heat was distributed through various layers from the backside of the IC die and how quickly the heat would dissipate into the IC substrate. Our modeling work explained the increase in signal from conductors through thicker dielectric layers and through polysilicon conductors between the laser source and the conductors of interest. The heat dissipation with time analyses indicated that a pulsed laser at a $1 \mathrm{MHz}$ pulse rate might provide increased signal sensitivity.

We attempted increasing the TIVA/SEI system's sensitivity by using a pulsed laser and a lock-in amplifier on the TIVA/SEI signal synchronized to the pulse frequency. This did not yield an improved defect signal as hoped. We believe that the minimum scan frequency of the scanning laser microscope is too fast to capture the TIVA/SEI signals. Another contributing factor may be an unexpected limitation in the power supply to react at the 1 $\mathrm{MHz}$ pulsed frequency used.

Milestone 9. Optimization of the system through increased thermal power to the sample was achieved by two actions. First, we used an approximately $4 \mathrm{X}$ higher power laser. Second, we inserted a $1 / 2$ wave plate into the beam path of the laser. By rotating the $1 / 2$ wave plate the polarization of the laser was rotated. The rotation of the plate could be set to minimize the reflection losses on the mirrors of the scanning laser microscope. Optimum placement of the $1 / 2$ wave plate increased the laser power delivered to the sample by about $50 \%$. The net result was a little more than $5 \mathrm{X}$ increase in power delivered to the sample.

Direct through metal examination has been limited to a single level, with some signal blur as the "point" heat source spreads laterally. No signal form the heat penetrating through more than one level has been observed. 
Milestone 10. and 11. The results of the second year's LDRD effort were presented at the European Symposium on Reliability of Electron Devices, Failure Physics and Analysis (ESREF) in October 1999. The paper was well received and the results of this work and the rest of the LDRD have led to much IC manufacturer as well as equipment vendor interest. Two licensing agreements with IC manufacturers are underway with additional licenses expected.

This document achieves the final milestone of a SAND report, three months after the September goal. We were granted an extension by the LDRD office due to travel by the principal investigator, Ed Cole.

Non-linear Effects. An unexpected bonus of the increase in laser power was a non-linear, almost exponential increase in SEI and TIVA signals with power. The increase occurs because the transistors on the IC associated with the heated defects respond as non-linear amplifiers. The $5 \mathrm{X}$ increase in laser power produce an almost 100X increase in SEI/TIVA signals on some devices. The result of these increases are greatly reduced analysis times (45 minutes to 16 seconds or less) and the ability to detect defects in less that 1 minute that could not be observed in the first year of the LDRD with 1 hour of signal averaging. Because of this increase in signal strength, the unsuccessful lock-in amplification approach for increasing the signal was not necessary for system optimization.

As this summary describes, we have met or surpassed almost all of our goals with the exception of the lock-in amplification to increase signal sensitivity. Fortunately, the non-linear increase in TIVA/SEI signals with laser power made the lock-in amplification unnecessary for system optimization. 


\section{PUBLICATIONS}

1. E.I. Cole Jr., P. Tangyunyong, and D.L. Barton, "Backside Localization of Open and Shorted Interconnections", $36^{\text {th }}$ Annual Proceedings of the International Reliability Physics Symposium, 129-136 (1998).

2. E.I. Cole Jr., P. Tangyunyong, and D.L. Barton, "Local Thermal Probing to Detect Open and Shorted IC Interconnections", Microelectronics Reliability, 39, 681-693, (1999). This was an invited paper for the journal.

3. E.I. Cole Jr., P. Tangyunyong, D.A. Benson, and D.L. Barton, "TIVA and SEI Developments for Enhanced Front and Backside Interconnection Failure Analysis", Proceedings of the $10^{\text {th }}$ European Symposium on Reliability of Electron Devices, Failure Physics and Analysis, 991996 (1999).

4. P. Tangyunyong, D.A. Benson, and E.I. Cole Jr., "Thermal Modeling of TIVA Profiles of a Polysilicon-Metal Test Structure", publication pending.

Workshops including TIVA/SEI analysis:

1. E.I. Cole Jr., "Advanced Techniques for Rapid Localization of Interconnect Defects", Advanced Metallization Conference.

This was an invited tutorial presentation.

2. D.L. Barton, K. Bernhard-Hofer, E.I. Cole Jr., "FLIP-chip and "Backside" Techniques," European Symposium on Reliability of Electron Devices, Failure Physics and Analysis.

This was an invited tutorial presentation.

3. D.L. Barton, K. Bernhard-Hofer, E.I. Cole Jr., "Backside Techniques," $25^{\text {th }}$ International Symposium for Testing and Failure Analysis.

\section{PATENTS AND INTELLECTUAL PROPERTY}

A patent application entitled "Thermally Induced Voltage Alteration for Integrated Circuits" was filed with the United States Patent and Trademark Office on March 3, 1998 and assigned Serial No. 09/034,546. The Sandia assigned number is SD-6101.

In August 1999, Ed Cole was contacted by a large commercial IC manufacturer expressing a strong interest in determining if TIVA/SEI would be applicable in their failure analysis laboratory. Subsequent testing and negotiations led to a licensing agreement that is now nearing completion.

Subsequent inquiries have been made by several other IC manufacturers concerning the acquisition of the TIVA/SEI technology in their laboratories. One license is currently under 
negotiation. Two companies have expressed interest in arranging a Work For Others (WFO) agreement with Sandia to test the applicability of TIVA and SEI to their product. Other interested parties are investigating their equipment needs for implementing the techniques.

\section{SYNERGISTIC WORK}

In April of 1998 Sandia initiated a CRADA for developing new failure analysis tools for advanced ICs. The tools developed as part of the CRADA have leveraged heavily on the developments of the TIVA/SEI project. The CRADA has been deemed successful by the customer, with the CRADA customer renewing the agreement for a second year. The CRADA has been a $\$ 300 \mathrm{~K}$ effort for both years, with $\$ 150 \mathrm{~K}$ in funds-in from the CRADA customer each year. Intellectual property has been generated as a result of the CRADA investigations and two joint patents are presently being prepared. The customer has expressed an interest in continuing work with Sandia for a $3^{\text {rd }}$ year in FY 01.

\section{POTENTIAL FUTURE WORK}

Several commercial IC vendors and equipment manufactures have expressed great interest in obtaining or producing a "turn-key" TIVA/SEI system. The interest is great enough that the SEMATECH Product Analysis Forum has issued a specification and proposal request for vendors to supply such a "turn-key" system. Sandia was involved in some of the details of the specification and would be licensing the vendors of any commercial TIVA/SEI systems produced. One possible vendor has already made a site visit to Sandia to discuss possible licensing options.

\section{BENEFITS TO DOE DEFENSE PROGRAMS}

The TIVA/SEI LDRD has benefited DOE Defense Programs by developing new technologies for non-invasive localization of open and shorted interconnections from the front and backside of an IC for failure analysis, yield improvement, and new process development. The technology is resident in Sandia National Laboratories Failure Analysis Department and has been used repeatedly for Stockpile Support and the development of new Sandia IC technologies. 


\section{DISTRIBUTION}

$\begin{array}{lrl}1 & \text { MS } 0188 & \text { LDRD Office, 4001 } \\ 1 & 0455 & \text { L.R. Gilliom, 6232 } \\ 1 & 1071 & \text { T.A.Dellin, 1700 } \\ 1 & 1074 & \text { P.V. Dressendorfer, 1726 } \\ 1 & 1078 & \text { P.J. McWhorter, 1702 } \\ 1 & 1079 & \text { D. Williams, 1700 } \\ 1 & 1081 & \text { R.E. Anderson, 1739 } \\ 1 & 1081 & \text { D.L. Barton, 1739 } \\ 5 & 1081 & \text { E.I. Cole Jr., 1739 } \\ 1 & 1081 & \text { P. Tangyunyong, 1739 } \\ 1 & 9018 & \text { Central Technical Files, 8940-2 } \\ 2 & 0899 & \text { Technical Library, 4916 } \\ 1 & 0612 & \text { Review and Approval Desk, 4912 } \\ 1 & & \text { For DOE/OSTI } \\ 1 & 0161 & \text { Patent and Licensing Office, 11500 }\end{array}$

